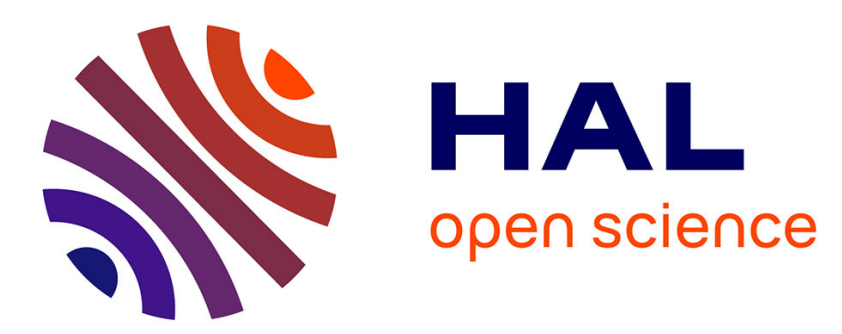

\title{
A fully coupled scheme using Virtual Element Method and Finite Volume for poroelasticity
}

Julien Coulet, Isabelle Faille, Vivette Girault, Nicolas Guy, Frédéric Nataf

\section{To cite this version:}

Julien Coulet, Isabelle Faille, Vivette Girault, Nicolas Guy, Frédéric Nataf. A fully coupled scheme using Virtual Element Method and Finite Volume for poroelasticity. Computational Geosciences, 2020, 24 (2), pp.381-403. 10.1007/s10596-019-09831-w . hal-01947455

\section{HAL Id: hal-01947455 \\ https://hal-ifp.archives-ouvertes.fr/hal-01947455}

Submitted on 6 Dec 2018

HAL is a multi-disciplinary open access archive for the deposit and dissemination of scientific research documents, whether they are published or not. The documents may come from teaching and research institutions in France or abroad, or from public or private research centers.
L'archive ouverte pluridisciplinaire HAL, est destinée au dépôt et à la diffusion de documents scientifiques de niveau recherche, publiés ou non, émanant des établissements d'enseignement et de recherche français ou étrangers, des laboratoires publics ou privés. 


\title{
A fully coupled scheme using Virtual Element Method and Finite Volume for poroelasticity
}

\author{
Julien Coulet · Isabelle Faille · Vivette Girault · Nicolas Guy · Frédéric Nataf
}

\begin{abstract}
In this paper, we design and study a fully coupled numerical scheme for the poroelasticity problem modelled through Biot's equations. The classical way to numerically solve this system is to use a finite element method for the mechanical equilibrium equation and a finite volume method for the fluid mass conservation equation. However, to capture specific properties of underground media such as heterogeneities, discontinuities and faults, meshing procedures commonly lead to badly shaped cells for finite element based modelling. Consequently, we investigate the use of the recent virtual element method which appears as a potential discretization method for the mechanical part and could therefore allow the use of a unique mesh for the both mechanical and fluid flow modelling. Starting from a first insight into virtual element method applied to the elastic problem in the context of geomechanical simulations, we apply in addition a finite volume method to take care of the fluid conservation equation. We focus on the first order virtual element method and the two point flux approximation for the finite volume part. A mathematical analysis of this original coupled scheme is provided, including existence and uniqueness results and a priori estimates. The method is then illustrated by some computations on two or three dimensional grids inspired by realistic application cases.
\end{abstract}

\footnotetext{
J. Coulet

IFP Energies nouvelles, 1-4 avenue de Bois-Préau, 92852 RueilMalmaison, France

Tel.: +33-147525404

E-mail: julien.coulet@ifpen.fr

I. Faille, N. Guy

IFP Energies nouvelles, 1-4 avenue de Bois-Préau, 92852 RueilMalmaison, France

V. Girault, F. Nataf

Sorbonne Université, Université Paris-Diderot SPC, CNRS, Laboratoire Jacques-Louis Lions, LJLL, F-75005 Paris
}

Keywords Virtual element methods - Finite volume methods · Polyhedral grids · Poroelasticity · Geomechanics · Reservoir simulation

Mathematics Subject Classification (2010) 65N30 . 74F10 $\cdot 76 \mathrm{~S} 05$

\section{Introduction}

Geomechanics relies on the description of the coupling between mechanical deformations of the media and subsurface flow. Over the past few decades, it became increasingly clear that taking into account the interaction between these two physics could give a better representation of the reality in certain cases such as reservoir simulation [30]. This led to coupled models and consequently raised the question of the numerical approximation of these models. The natural choice has been to use two spatial discretization methods, each being widely used for one of the two physical problems: finite element method for the mechanical part and finite volume method for the flow part [25]. To work properly, this approach requires either a mesh compatible with the two discretization methods, or two distinct meshes with some interpolation operators to transfer the variables from one to the other, see $e g$ [20]. The point is that when dealing with subsurface media, the specific geometries encountered such as heterogeneities or faults make it difficult to obtain a reasonable mesh admissible for the finite element method.

For these reasons, we are interested in replacing the finite element method by another numerical scheme that allows for more general meshes. Our aim is to use it together with the finite volume scheme on the same spatial grid. Several such schemes have been identified in recent reviews [8]. Without being exhaustive, we may mention the extension of finite element methods to polyhedral meshes with the use of barycentric [27] or harmonic [11] shape func- 
tions, the families of Discontinuous Galerkin [24], Hybrid Discontinuous Galerkin [15] and Hybrid High Order [16] methods, the Weak Galerkin method [29] or the Mimetic Finite Differences (MDF) [28]. This last category, rewritten in a variational framework, gave birth to the Virtual Element Method (VEM) which allows very general cells geometries [22]. Compared to the forementioned methods, VEM avoid manipulating cumbersome polynomial shape functions or managing a large number of degrees of freedom. They benefit from a theoretical framework inherited from the MFD and enriched by some works covering for example stability [9], a priori [12] and a posteriori [14] estimates or serendipity [7]. From their original introduction in [3], these methods have been applied to an increasing number of problems, including linear elasticity in two or three dimensions, even in the specific context of geomechanical simulations [2].

The aim of our work is to design and study a coupled numerical scheme for poroelastic problems using this virtual element method formulation for the mechanical equilibrium together with a finite volume scheme applied to the fluid conservation equation. This last choice is motivated by the existence of robust finite volume schemes suitable for the discretization of the flow problem over badly shaped grids. In addition, these schemes guarantee the fluid mass conservation on contrast to the virtual element methods.

This paper is thus organized in the following way. Section 2 recalls the poroelastic model to be solved. The construction of the coupled scheme is detailed in section 3. A certain generality on the choice of the two discretization methods is initially kept, but we then focus on the use of the lowest order virtual element method and of the simplest finite volume scheme. This choice of underlying methods allows us to provide a mathematical analysis of the coupled scheme in section 4. Existence and uniqueness results are established as well as an a priori error estimate. We show that in a certain norm, the convergence rate of the coupled scheme is one in time and in space. Section 5 illustrates the behaviour of the scheme, first on an academic case in order to confirm the theoretical part, and then on a realistic physical case.

\section{The poroelastic model}

The governing equations of the interactions between mechanical deformations and fluid flow in a porous media result from the work of [10]. The modelling involves two coupled equations obtained from the mechanical equilibrium and from the fluid mass conservation, under the assumptions of quasistatic strains and slightly compressible single-phase flow.

Given an open subset $\Omega$ of $\mathbb{R}^{d}(d=2$ or 3 ) with boundary $\partial \Omega$ and unit outward normal $\mathbf{n}$, let $\mathbf{u}(\mathbf{x}, t)$ and $p(\mathbf{x}, t)$ respectively refer to the displacement vector and to the fluid pressure. For a volumetric force $\mathbf{f}: \Omega \times] 0, T] \rightarrow \mathbb{R}^{d}$, the mechanical equilibrium reads

$-\operatorname{div}\left(\bar{\sigma}^{e}(\mathbf{u})-\alpha p \overline{I_{d}}\right)=\mathbf{f}$

where the total stress $\bar{\sigma}(\mathbf{u}, p):=\bar{\sigma}^{e}(\mathbf{u})-\alpha p \overline{I_{d}}$ depends on the effective stress $\bar{\sigma}^{e}(\mathbf{u})$ and on the pressure through the dimensionless Biot-Willis coefficient $\alpha$ close to one. The mechanics sign convention is adopted for the stresses. When dealing with linear elastic solids, the effective stress is given by the classical relation

$$
\bar{\sigma}^{e}(\mathbf{u})=\overline{\bar{C}} \bar{\varepsilon}(\mathbf{u}) \text { with } \bar{\varepsilon}=\frac{1}{2}\left(\overline{\nabla \mathbf{u}}+\overline{\nabla \mathbf{u}}^{T}\right)
$$

where $\overline{\bar{C}}$ is the fourth-order stiffness tensor which, in the simplest case of isotropic elasticity, can be described by two independent piecewise constant coefficients such as Young modulus $E$ and Poisson coefficient $v$.

The mass conservation equation is applied to the fluid content $c_{0} p+\alpha \operatorname{div}(\mathbf{u})$ taking into account the mechanical variation of pore volume $\alpha \operatorname{div}(\mathbf{u})$ and the constrained specific storage coefficient $c_{0}>0$. This last coefficient, whose dimension is the inverse of a pressure, measures the amount of fluid that can be injected in the media during a pressure increase at constant pore volume. The fluid velocity $\mathbf{v}_{f}$ is given by Darcy's law

$\mathbf{v}_{f}=-\bar{\kappa}\left(\nabla p-\rho_{f} \mathbf{g}\right)$,

$\mathbf{g}$ being the gravity field, which means that $\mathbf{v}_{f}$ is proportional to the hydrostatic disequilibrium with a $d \times d$ matrix ratio $\bar{\kappa}$ called mobility matrix. This matrix is defined as the quotient of the media permeability and the fluid dynamic viscosity. Under the slightly compressible hypothesis, the mass conservation equation leads to the volume conservation equation

$\partial_{t}\left(c_{0} p+\alpha \operatorname{div}(\mathbf{u})\right)+\operatorname{div}\left(-\bar{\kappa}\left(\nabla p-\rho_{f} \mathbf{g}\right)\right)=q$

for any volumetric source term $q: \Omega \times] 0, T] \rightarrow \mathbb{R}$.

The conservation relations (1) and (2) are complemented by initial and boundary conditions applied to both equations to form the poroelastic system : assuming that $\partial \Omega=\Gamma_{D_{d}} \cup$ $\Gamma_{N_{d}}, \Gamma_{D_{d}} \cap \Gamma_{N_{d}}=\emptyset$ and $\left|\Gamma_{D_{d}}\right|>0$ (resp. $\Gamma_{D_{p}}, \Gamma_{N_{p}}$ ) and given $\left.\left.\left.\left.\mathbf{t}_{N}: \Gamma_{N_{d}} \times\right] 0, T\right] \rightarrow \mathbb{R}^{d}, \phi_{N}: \Gamma_{N_{p}} \times\right] 0, T\right] \rightarrow \mathbb{R}$ and $p^{0}: \Omega \rightarrow \mathbb{R}$ the pair $(\mathbf{u}, p)$ satisfies

in $\Omega \times] 0, T]$ :

$\begin{aligned}-\operatorname{div}\left(\overline{\bar{C}} \bar{\varepsilon}_{u}-\alpha p \overline{I_{d}}\right) & =\mathbf{f}, \\ \partial_{t}\left(c_{0} p+\alpha \operatorname{div}(\mathbf{u})\right)+\operatorname{div}\left(-\bar{\kappa}\left(\nabla p-\rho_{f} \mathbf{g}\right)\right) & =q,\end{aligned}$ 
on the boundaries for $t \in] 0, T]$ :

$$
\begin{array}{r}
\mathbf{u}=\mathbf{0} \text { on } \Gamma_{D_{d}}, \\
\left(\overline{\sigma^{e}}-\alpha p \overline{I_{d}}\right) \cdot \mathbf{n}=\mathbf{t}_{N} \text { on } \Gamma_{N_{d}}, \\
p=0 \text { on } \Gamma_{D_{p}}, \\
-\bar{\kappa}\left(\nabla p-\rho_{f} \mathbf{g}\right) \cdot \mathbf{n}=\phi_{N} \text { on } \Gamma_{N_{p}},
\end{array}
$$

and for $\mathbf{x} \in \Omega$ at initial time:

$$
\begin{aligned}
p(\mathbf{x}, t=0) & =p^{0}(\mathbf{x}), \\
\mathbf{u}(\mathbf{x}, t=0) \text { solution of (3a) with } p & =p^{0} .
\end{aligned}
$$

Existence and uniqueness results of a continuous solution of (3), under certain regularity requirement on the data, can be found in [26].

\section{Construction of the coupled discretization}

This section starts with a generic formulation using a variational approximation of the mechanical equation and a finite volume formulation of the flow equation. Some points, as the approximation spaces or the exact discrete operators, are not specified at this stage. This allows to substitute afterward any VEM-like operator and any finite volume operator in the coupled scheme. With this in mind, subsection 2 describes the construction of the first order virtual element method operator for the elastic problem, whereas subsection 3 briefly recalls a finite volume approximation for the fluid problem. The last subsection reverts to the coupled scheme; it uses these two discretizations and adds a few details on the treatment of coupling terms. For the sake of simplicity, the gravity term is omitted.

\subsection{The generic coupled scheme}

Let $V_{0}$ and $Q$ be the following function spaces:

$V_{0}=\left\{\mathbf{v} \in\left(H^{1}(\Omega)\right)^{d}: \quad \mathbf{v}_{\mid \Gamma_{D_{d}}}=\mathbf{0}\right\}$

$Q=\left\{p \in H^{1}(\Omega): \quad p_{\mid \Gamma_{D_{p}}}=0\right\}$.

Let $\mathbf{f} \in\left(L^{2}(\Omega \times] 0, T[)\right)^{d}$ and $\mathbf{t}_{N} \in\left(L^{2}\left(\Gamma_{N_{d}} \times\right] 0, T[)\right)^{d}$. For the purpose we have in mind, on the one hand, the elasticity equation is set into a variational form by integrating ( $3 a$ ) by parts against any test function $\mathbf{v}$ of $V_{0}$,

$\int_{\Omega} \overline{\bar{C}} \overline{\varepsilon_{u}}: \overline{\varepsilon_{v}}-\int_{\Omega} \alpha p \operatorname{div}(\mathbf{v})=\int_{\Omega} \mathbf{f} \cdot \mathbf{v}+\int_{\Gamma_{N_{d}}} \mathbf{t}_{N} \cdot \mathbf{v}$.

We recognize above the symmetric continuous bilinear coercive form on $V_{0}$ associated to the standard elasticity problem,

$a^{\Omega}(\mathbf{u}, \mathbf{v}):=\int_{\Omega} \overline{\bar{C}} \overline{\varepsilon_{u}}: \overline{\varepsilon_{v}}$.
On the other hand, the variational formulation for the flow equation will be replaced below by a suitable integration on control volumes.

Now, we turn to the discretization. The domain $\Omega$ is decomposed into non overlapping Lipschitz polyhedra $K_{i}$ such that $\stackrel{\circ}{K}_{i} \cap \stackrel{\circ}{K}_{j}=\emptyset$ and $\cup_{i} K_{i}=\bar{\Omega}$. For each polyhedron, $h_{K}$ is the diameter of $K,|K|$ its measure and $\mathbf{x}^{K}$ its barycenter. Its set of vertex indices is denoted $\mathscr{M}_{K}$ and its number of vertices is denoted $M_{K}$. In the three dimensional case, we use the superscript $f$ to similarly define the quantities $h_{f},|f|, \mathbf{x}^{f}, \mathscr{M}_{f}$ and $M_{f}$ for each face $f$ of $K$. In the two dimensional case, we keep the notation $K$ to designate the polygons. This tessellation of $\Omega$ is denoted $\tau_{h}$ where $h=$ $\max _{K}\left(h_{K}\right)$. Let $\left(V_{h, 0}, Q_{h}\right)$ be a pair of discrete spaces and let $a_{h}$ be a discrete approximation of the bilinear form $a^{\Omega}$, to be specified further on. The space approximation of (4) consists in searching for $\left(\mathbf{u}_{h}, p_{h}\right)$ in $\left(V_{h, 0}, Q_{h}\right)$ satisfying

$a_{h}\left(\mathbf{u}_{h}, \mathbf{v}_{h}\right)-\sum_{K} \alpha \int_{K} \operatorname{div}\left(\mathbf{v}_{h}\right) p_{h}=\int_{\Omega} \mathbf{f} \cdot \mathbf{v}_{h}+\int_{\Gamma_{N_{d}}} \mathbf{t}_{N} \cdot \mathbf{v}_{h}$

$\forall \mathbf{v}_{h} \in V_{h, 0}$. Concerning the fluid equation, Stokes formula is used on each cell $K$ to write

$$
\begin{aligned}
\forall K \in \tau_{h}, \quad \int_{K} \partial_{t}\left(c_{0} p+\alpha \operatorname{div}(\mathbf{u})\right) & +\int_{\partial K \backslash \Gamma_{N_{p}}}-\bar{\kappa} \nabla p \cdot \mathbf{n}_{K} \\
& =\int_{K} q-\int_{\partial K \cap \Gamma_{N_{p}}} \phi_{N}
\end{aligned}
$$

where $\mathbf{n}_{K}$ is the outward unit normal to $K$. In order to discretize the fluid equation (7), the time interval $[0, T]$ is split into time steps $\left(t^{n}\right)_{n=0}^{N}$ such that $\Delta t:=t^{n+1}-t^{n}$ is constant. A backward Euler scheme is used to approximate the time derivative: denoting $\zeta^{n}(\mathbf{x})=\zeta\left(\mathbf{x}, t^{n}\right)$,

$\partial_{t} \zeta^{n}(\mathbf{x}) \approx D^{-} \zeta^{n}(\mathbf{x}):=\frac{\zeta^{n}(\mathbf{x})-\zeta^{n-1}(\mathbf{x})}{\Delta t}$

This time discretization is applied to equation (7), and following the finite volume framework, see [18], the boundary integral $\int_{f \subset \partial K}-\bar{\kappa} \nabla p \cdot \mathbf{n}$ leaving cell $K$ across face $f$ is approximated by a numerical flux $\mathscr{F}_{K f}$ to obtain, for each cell $K \in \tau_{h}$ and for every $n, 1 \leq n \leq N$,

$$
\begin{aligned}
& \int_{K}\left(c_{0} p_{h}^{n}+\alpha \operatorname{div}\left(\mathbf{u}_{h}^{n}\right)\right)+\Delta t \sum_{\substack{f \subset \partial K \\
f \not \subset \Gamma_{N_{p}}}} \mathscr{F}_{K f}^{n}= \\
& \int_{K}\left(\Delta t q^{n}+c_{0} p_{h}^{n-1}+\alpha \operatorname{div}\left(\mathbf{u}_{h}^{n-1}\right)\right)-\Delta t \int_{\partial K \cap \Gamma_{N_{p}}} \phi_{N}^{n} .
\end{aligned}
$$

The generic discrete coupled scheme is therefore given by: 


\section{Generic VEM-FV formulation}

For each time $t^{n}$, find $\left(\mathbf{u}_{h}^{n}, p_{h}^{n}\right) \in\left(V_{h, 0} \times Q_{h}\right)$ such that

$\forall \mathbf{v}_{\mathbf{h}} \in V_{h, 0}$,

$a_{h}\left(\mathbf{u}_{h}^{n}, \mathbf{v}_{h}\right)-\sum_{K} \alpha \int_{K} \operatorname{div}\left(\mathbf{v}_{h}\right) p_{h}^{n}=\int_{\Omega} \mathbf{f}^{n} \cdot \mathbf{v}_{h}+\int_{\Gamma_{N_{d}}} \mathbf{t}_{N}^{n} \cdot \mathbf{v}_{h}$

$\forall K \in \tau_{h}, \quad \int_{K}\left(c_{0} p_{h}^{n}+\alpha \operatorname{div}\left(\mathbf{u}_{h}^{n}\right)\right)+\Delta t \sum_{\substack{f \subset \partial K \\ f \not \subset \Gamma_{N}}} \mathscr{F}_{K f}^{n}$

$=\int_{K}\left(\Delta t q^{n}+c_{0} p_{h}^{n-1}+\alpha \operatorname{div}\left(\mathbf{u}_{h}^{n-1}\right)\right)-\Delta t \int_{\partial K \cap \Gamma_{N_{p}}} \phi_{N}^{n}$

We will now first construct the first order virtual element method used for the elasticity equation, defining the space $V_{h, 0}$ and the discrete form $a_{h}$, and secondly choose a finite volume scheme to be used for the fluid equation, defining the space $Q_{h}$ and the numerical flux $\mathscr{F}_{K f}$. From now, we use the following notations for the norms: in a region $\omega$,

$\|v\|_{L^{2}(\omega)}=\left(\int_{\omega} v^{2}\right)^{\frac{1}{2}},\|v\|_{H^{1}(\omega)}=\left(\int_{\omega} v^{2}+\nabla v \cdot \nabla v\right)^{\frac{1}{2}}$

and

$\|\mathbf{v}\|_{L^{2}(\omega)}=\left(\int_{\omega} \mathbf{v} \cdot \mathbf{v}\right)^{\frac{1}{2}},\|\mathbf{v}\|_{H^{1}(\omega)}=\left(\int_{\omega} \mathbf{v} \cdot \mathbf{v}+\nabla \mathbf{v}: \nabla \mathbf{v}\right)^{\frac{1}{2}}$.

For more readability, we will just write $\|\cdot\|_{L^{2}}$ or $\|\cdot\|_{H^{1}}$ when $\omega \equiv \Omega$. For the time discretization, we also define the norms

$\|v\|_{L^{2}(T)}=\left(\int_{t^{0}}^{t^{N}} v(t)^{2} d t\right)^{\frac{1}{2}}$ and $\|v\|_{L^{\infty}(T)}=\max _{n \in[0, N]}\left|v\left(t^{n}\right)\right|$.

These spatial and in time norms may be compacted in double subscript such as

$\|v\|_{L^{2}\left(0, T ; H^{1}\right)}=\|v\|_{L^{2}\left(0, T ; H^{1}(\Omega)\right)}=\left(\int_{t^{0}}^{t^{N}}\|v(t)\|_{H^{1}(\Omega)}^{2} d t\right)^{\frac{1}{2}}$.

3.2 Virtual element method for the elasticity equation

In this part, we focus on the elasticity equation

Find $\mathbf{u} \in V_{0}$ s.t. $\forall \mathbf{v} \in V_{0}$,

$$
a^{\Omega}(\mathbf{u}, \mathbf{v})=\int_{\Omega} \mathbf{f} \cdot \mathbf{v}+\int_{\Gamma_{N_{d}}} \mathbf{t}_{N} \cdot \mathbf{v}:=l(v)
$$

and we introduce the core ideas of the first order virtual element method. For more details, see eg [6] for theoretical aspects or [19] for implementation aspects.
Approximation space On each element $K$, an approximation space $V_{h}^{K} \subset H^{1}(K)$ is built in a such way that

- The functions of $V_{h}^{K}$ are well-defined at the vertices of $K$.

- First order polynomial functions are included in $V_{h}^{K}$.

- Any function in $V_{h}^{K}$ is determined by its values at the $M_{K}$ vertices of $K$.

- In three dimensions (respectively, two dimensions), the trace of any function of $V_{h}^{K}$ on any face $f$ (respectively, edge $e$ ) of the boundary $\partial K$ of $K$ depends only on the values of the function at the vertices of $f$ (respectively, $e)$.

Thus the degrees of freedom of $V_{h}^{K}$ are the values of its functions at the vertices of $K$. Furthermore, as in standard finite element theory, the last property above guarantees that the spaces $V_{h}^{K}$ can be assembled so as to constitute a conforming approximation of $H^{1}(\Omega)$. We start with the two dimensional case: $K$ thus stands for a given polygon. The scalar space $V_{h, s c a l}^{K}$ defined as follows satisfies these conditions:

$$
\begin{array}{r}
V_{h, s c a l}^{K}=\left\{v_{h} \in H^{1}(K): \forall \text { edge } e \in \partial K, v_{h \mid e} \in \mathbb{P}_{1}(e) ;\right. \\
\left.v_{h \mid \partial K} \in \mathscr{C}^{0}(\partial K) ; \quad \Delta v_{h}=0 \text { in } K\right\} .
\end{array}
$$

In three dimensions, the definition of $V_{h, s c a l}^{K}$ is less obvious. The notation $K$ now stands for a polyhedron made of polygonal faces $f$. We first define on each of these faces an operator $\pi^{f, 0}:\left\{v \in H^{1}(f): v \in \mathscr{C}^{0}(\partial f)\right\} \rightarrow \mathbb{P}_{1}$ by

$\pi^{f, 0} v(\mathbf{x})=\langle\nabla v\rangle\left(\mathbf{x}-\overline{\mathbf{x}_{f}}\right)+\overline{v_{f}}$

where $\langle\nabla v\rangle=\frac{1}{|f|} \int_{f} \nabla v$ and $\overline{\zeta_{f}}=\frac{1}{M_{f}} \sum_{i \in \mathscr{M}_{f}} \zeta(V i)$ with $\zeta \equiv$ $\mathbf{x}$ or $\zeta \equiv v$. We now build on each face a space in which $\pi^{f, 0}$ is a $L^{2}$ projection:

$$
\begin{array}{r}
V_{h, s c a l}^{f}=\left\{v_{h} \in H^{1}(f): \forall e, v_{h \mid e} \in \mathbb{P}_{1}(e) ; v_{h \mid \partial f} \in \mathscr{C}^{0}(\partial f) ;\right. \\
\left.\Delta v_{h} \in \mathbb{P}_{1} \text { in } f ; \quad \int_{f} \pi^{f, 0} v_{h} q=\int_{f} v_{h} q \forall q \in \mathbb{P}_{1}\right\} .
\end{array}
$$

Finally, the three dimensional approximation space on the polyhedron $V_{h, s c a l}^{K}$ is constructed from the polygonal spaces by

$$
\begin{array}{r}
V_{h, s c a l}^{K}=\left\{v_{h} \in H^{1}(K): v_{h \mid f} \in V_{h, s c a l}^{f} \forall f \in \partial K ;\right. \\
\left.\Delta v_{h}=0 \text { in } K\right\} .
\end{array}
$$

Remark: the natural extension to three dimensions would have been to simply use the definition (12) with $K=f$ for the polygonal space $V_{h, s c a l}^{f}$. Unfortunately, this choice lead to an approximation space that contains less informations and does not allow to evaluate exactly the bilinear form. Nevertheless, the two polygonal spaces are equivalent in term of dimension and degrees of freedom as shown in [1]. 
In both cases, we note that the approximation space is reduced to $\mathbb{P}_{1}$ on simplicial cells. In the general case, additional functions are contained in $V_{h, s c a l}^{K}$. The fact that the explicit knowledge of these functions is not required justifies the terminology virtual and accounts for the flexibility of the method. The global scalar space $V_{h, s c a l}$ then comes from the conformal assembling of the local spaces

$V_{h, s c a l}=\left\{v_{h} \in H^{1}(\Omega): v_{h \mid K} \in V_{h, s c a l}^{K}\right.$ for all $\left.K \in \tau_{h}\right\}$.

The global degrees of freedom of $V_{h, s c a l}$ are the values of functions at the $M$ vertices of $\tau_{h}$ : for each vertex $V_{i}$, the scalar basis function is defined by $\varphi_{i}\left(V_{j}\right)=\delta_{i j} \forall j \in \mathscr{M}$ leading to the Lagrange interpolation identity

$\forall v_{h} \in V_{h, s c a l}, \quad v_{h}(\mathbf{x})=\sum_{i \in \mathscr{M}} v_{h}\left(V_{i}\right) \varphi_{i}(\mathbf{x})$

where $\mathscr{M}$ denotes the set of vertex indices. In the case of our vectorial problem, the scalar spaces are duplicated to construct the approximation spaces $V_{h}^{K}=\left(V_{h, s c a l}^{K}\right)^{d}$ and $V_{h}=$ $\left(V_{h, s c a l}\right)^{d}$. The scalar basis functions are used to define $d$ basis functions on each node by $\varphi_{i}^{k}=\varphi_{i} \mathbf{e}_{k}(k=x, y, z)$. The functions belonging to $V_{h}$ are expressed on the $\left\{\varphi_{i}^{k}\right\}$ basis by

$\mathbf{v}_{h}(\mathbf{x})=\sum_{i \in \mathscr{M}} \mathbf{v}_{h}^{x}\left(V_{i}\right) \varphi_{i}^{x}(\mathbf{x})+\mathbf{v}_{h}^{y}\left(V_{i}\right) \varphi_{i}^{y}(\mathbf{x})+\mathbf{v}_{h}^{z}\left(V_{i}\right) \varphi_{i}^{z}(\mathbf{x})$.

Approximate weak formulation Because the exact bilinear form can not be directly computed for the virtual basis functions, the VEM strategy is to replace it by an approximate bilinear form $a_{h}$ chosen to be computable from the degrees of freedom. This is done element wise by using the splitting

$a^{\Omega}\left(\mathbf{u}_{h}, \mathbf{v}_{h}\right)=\sum_{K \in \tau_{h}} a^{K}\left(\mathbf{u}_{h}, \mathbf{v}_{h}\right)$ where $a^{K}(\mathbf{u}, \mathbf{v})=\int_{K} \overline{\bar{C}} \overline{\varepsilon_{u}}: \overline{\varepsilon_{v}}$,

approximating $a_{h}^{K}\left(\mathbf{u}_{h}, \mathbf{v}_{h}\right) \approx a^{K}\left(\mathbf{u}_{h}, \mathbf{v}_{h}\right)$ in each element, and building $a_{h}=\sum_{K \in \tau_{h}} a_{h}^{K}$. Despite this variational crime, convergence remains ensured when the two following properties are verified:

1. Consistency: for all $\mathbf{p} \in \mathbb{P}_{1}^{d}$, for all $\mathbf{v}_{h} \in V_{h}^{K}$

$a_{h}^{K}\left(\mathbf{v}_{h}, \mathbf{p}\right)=a^{K}\left(\mathbf{v}_{h}, \mathbf{p}\right)$.

This property ensures the accuracy of the method. It allows the patch test to be satisfied, in the sense that the method is exact when the true solution is linear.

2. Stability: there exist two positive constants $\alpha_{\star}, \alpha^{\star}$ independent of $K$ and $h$ such that $\forall \mathbf{v}_{h} \in V_{h}^{K}$,

$\alpha_{\star} a^{K}\left(\mathbf{v}_{h}, \mathbf{v}_{h}\right) \leq a_{h}^{K}\left(\mathbf{v}_{h}, \mathbf{v}_{h}\right) \leq \alpha^{\star} a^{K}\left(\mathbf{v}_{h}, \mathbf{v}_{h}\right)$

This inequality guarantees the coercivity of the form $a_{h}$ and the uniqueness of the solution.
Since property (15) applies to the polynomial part of the functions of $V_{h}^{K}$, defining a local projection operator $\pi^{K}$ from $V_{h}^{K}$ to $\mathbb{P}_{1}^{d}$ to ensure the consistency seems to be a good idea. In fact, it is easy to see that if

$\forall \mathbf{v}_{h} \in V_{h}^{K}, \quad a^{K}\left(\pi^{K} \mathbf{v}_{h}, \mathbf{q}\right)=a^{K}\left(\mathbf{v}_{h}, \mathbf{q}\right)$ for all $\mathbf{q} \in \mathbb{P}_{1}^{d}$,

the approximate form $\tilde{a}_{h}^{K}(\mathbf{u}, \mathbf{v}):=a^{K}\left(\pi^{K} \mathbf{u}, \pi^{K} \mathbf{v}\right)$ is consistent (using $\pi^{K} \mathbf{p}=\mathbf{p}$ for $\mathbf{p} \in \mathbb{P}_{1}^{d}$ ). Moreover, we will show later that such a projector can be computed from degrees of freedom even for the virtual functions. At this point, we shall remark that $\tilde{a}_{h}^{K}$ does not respect the stability property (16). Following [6], the addition of the bilinear form

$\tilde{s}_{h}^{K}(\mathbf{u}, \mathbf{v}):=h_{K}^{d-2} \overline{\bar{C}}_{\infty} \sum_{i \in \mathscr{M}_{K}}\left(\mathbf{u}-\pi^{K} \mathbf{u}\right)\left(V_{i}\right) \cdot\left(\mathbf{v}-\pi^{K} \mathbf{v}\right)\left(V_{i}\right)$,

where $\overline{\bar{C}}_{\infty}=\max _{i j k l}\left|\overline{\bar{C}}_{i j k l}\right|$, allows to recover the stability property without affecting the consistency. Consequently, the sum of $\tilde{a}_{h}^{K}$ and $\tilde{s}_{h}^{K}$ defines the consistent and stable approximate bilinear form

$$
\begin{aligned}
& a_{h}^{K}(\mathbf{u}, \mathbf{v}):=a^{K}\left(\pi^{K} \mathbf{u}, \pi^{K} \mathbf{v}\right) \\
& \quad+h_{K}^{d-2} \max |\overline{\bar{C}}| \sum_{i \in \mathscr{M}_{K}}\left(\mathbf{u}-\pi^{K} \mathbf{u}\right)\left(V_{i}\right) \cdot\left(\mathbf{v}-\pi^{K} \mathbf{v}\right)\left(V_{i}\right) .
\end{aligned}
$$

For the same reasons, the right-hand side of the variational formulation has to be approximated by a computable discrete linear form $l_{h}\left(\mathbf{v}_{h}\right)$. Proceeding again element-wise, the integrals are approximated by

$l_{h}^{K}\left(\mathbf{v}_{h}\right):=\sum_{i \in \mathscr{M}_{K}} \omega_{i}^{K} \mathbf{f}\left(V_{i}\right) \cdot \mathbf{v}_{h}\left(V_{i}\right)+\sum_{i \in \mathscr{M}_{K} \cap \Gamma_{N_{d}}} \omega_{i}^{\partial K} \mathbf{t}_{N}\left(V_{i}\right) \cdot \mathbf{v}_{h}\left(V_{i}\right)$

where $\omega_{i}^{K}$ and $\omega_{i}^{\partial K}$ are dimension dependent integration weights, taken from [19] and illustrated by figure 1 . The order of the error due to this quadrature approximation is equivalent to the one produced by the numerical scheme. Other choices are possible for this load term approximation, see $e g$ [2]. We then construct $l_{h}=\sum_{K \in \tau_{h}} l_{h}^{K}$.

Let $\mathscr{M}^{\text {int }}$ be the set of non Dirichlet degrees of freedom indices (including Neumann boundary), and let $M^{\text {int }}$ denote the number of these indices. Introducing the space

$V_{h, 0}=\left\{\mathbf{v}_{h} \in V_{h}: \mathbf{v}_{h}\left(V_{i}\right)=\mathbf{0}\right.$ if $\left.i \notin \mathscr{M}^{\text {int }}\right\}$

and testing the unknown $\mathbf{u}_{h}$ against each basis function, the approximate variational formulation of (11) reads

\section{Approximate variational problem}

Find $\mathbf{u}_{h} \in V_{h, 0}$ such that for all $\mathbf{v}_{h} \in V_{h, 0}$,

$a_{h}\left(\mathbf{u}_{h}, \mathbf{v}_{h}\right)=l_{h}\left(\mathbf{v}_{h}\right)$

or equivalently, find $\mathscr{U}=\left(u_{h 1}^{x}, u_{h 1}^{y}, \cdots, u_{h M^{\text {int }}}^{z}\right)$ solution of the linear system

$\mathscr{A}_{h} \mathscr{U}=\mathscr{L}^{u}$

with $\mathscr{A}_{h i_{k} j_{l}}=a_{h}\left(\varphi_{i}^{k}, \varphi_{j}^{l}\right)$ and $\mathscr{L}_{i_{k}}^{u}=l_{h}\left(\varphi_{i}^{k}\right)$. 


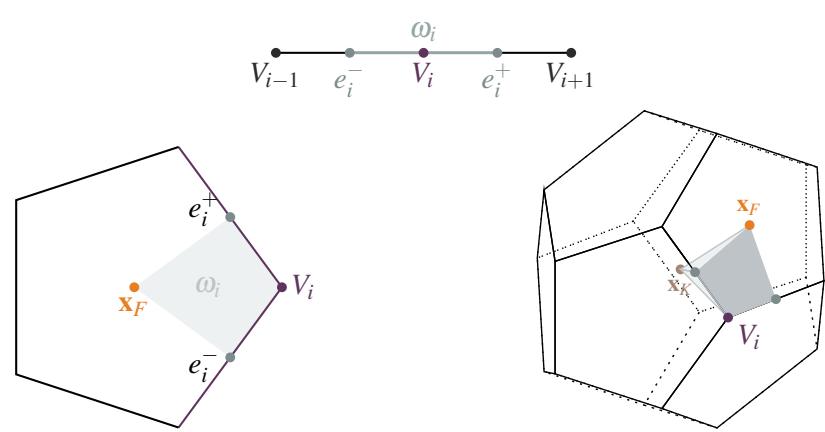

Fig. 1 Integration weight using node values for the right-hand side. In one dimension, $\omega_{i}$ is the length of the line connecting the midpoints $e_{i}^{-}, e_{j}^{+}$. In two dimensions, $\omega_{i}$ is the area of the quadrangle with vertices $V_{i}$, the barycenter $\mathbf{x}^{F}$ and the line midpoints $e_{i}^{-}, e_{i}^{+}$. In three dimensions, $\omega_{i}$ is the volume of the three pyramids based on $V_{i}, \mathbf{x}^{F}$, midpoints $e_{i}$ and having $\mathbf{x}^{K}$ (barycenter of $K$ ) as summit. For more readability, only one of these three pyramids is shown on the sketch.

This problem admits a unique solution satisfying the error estimate of Lemma 1, as shown in [4].

Lemma 1 Assume that each cell $K$ is star shaped with respect to a ball of radius $r h_{K}$, where $r>0$ is a constant independent of $h$ and $K$, smaller than the ratio between the shortest edge of $K$ and its diameter. Under the consistency (15) and stability (16) properties, the approximate problem (20a) has a unique solution $\mathbf{u}_{h}$. Moreover, for every approximation $\mathbf{u}_{I}$ of $\mathbf{u}$ in $V_{h}$ and for every approximation $\mathbf{u}_{\pi}$ of $\mathbf{u}$ that is piecewise in $\mathbb{P}_{1}^{d}$,

$$
\begin{aligned}
\left\|\mathbf{u}_{h}-\mathbf{u}\right\|_{H^{1}} \leq C\left(\left\|\mathbf{u}-\mathbf{u}_{I}\right\|_{H^{1}}\right. & +\left(\sum_{K \in \tau_{h}}\left\|\mathbf{u}-\mathbf{u}_{\pi}\right\|_{H^{1}(K)}^{2}\right)^{\frac{1}{2}} \\
& \left.+\sup _{\mathbf{v}_{h} \in V_{h, 0}} \frac{l\left(\mathbf{v}_{h}\right)-l_{h}\left(\mathbf{v}_{h}\right)}{\left\|\mathbf{v}_{h}\right\|_{H^{1}}}\right)
\end{aligned}
$$

where $C$ is a constant depending only on $\Omega, \overline{\bar{C}}, \alpha^{\star}, \alpha_{\star}$ and on the coercivity constant of $a$.

The assembling of the equivalent linear system is done element wise following the classical strategy of finite element methods. The only difficulty is the computation of the projection $\pi^{K}\left(\varphi_{i}^{k}\right)$ for each basis function $\varphi_{i}^{k}$ in order to evaluate $a_{h}$.

Computing $\pi^{K}$ The general method to compute the projection $\pi^{K}$ is to explicit the system (17) for each basis function and to solve it, obtaining the matrix representation of $\pi^{K}$ as done in [5]. However, at the lowest order of consistency $\left(\mathbf{p} \in \mathbb{P}_{1}^{d}\right.$ in (15)), it can be shown that the projector explicitly defined below by (21) satisfies the condition (17):

$\pi^{K} \mathbf{v}(\mathbf{x})=\langle\nabla \mathbf{v}\rangle\left(\mathbf{x}-\overline{\mathbf{x}_{K}}\right)+\overline{\mathbf{v}_{K}}$

where $\langle\nabla \mathbf{v}\rangle=\frac{1}{|K|} \int_{K} \nabla \mathbf{v}$ and $\overline{\zeta_{K}}=\frac{1}{M_{K}} \sum_{i \in \mathscr{M}_{K}} \zeta(V i)$ with $\zeta \equiv$ $\mathbf{x}$ or $\zeta \equiv \mathbf{v}$.
Indeed, using the fact that $\bar{\varepsilon}(\mathbf{p})$ is constant for all $\mathbf{p} \in \mathbb{P}_{1}^{d}$ and recalling that $\overline{\bar{C}}$ is piecewise constant,

$$
\begin{aligned}
a^{K}\left(\pi^{K} \mathbf{v}_{h}-\mathbf{v}_{h}, \mathbf{p}\right) & =\int_{K} \bar{\varepsilon}\left(\pi^{K} \mathbf{v}_{h}-\mathbf{v}_{h}\right): \overline{\bar{C}} \bar{\varepsilon}(\mathbf{p}) \\
& =\overline{\bar{C}} \bar{\varepsilon}(\mathbf{p}): \int_{K} \bar{\varepsilon}\left(\pi^{K} \mathbf{v}_{h}-\mathbf{v}_{h}\right)
\end{aligned}
$$

and using the above definition of $\pi^{K}$, we have

$\bar{\varepsilon}\left(\pi^{K} \mathbf{v}_{h}\right)-\bar{\varepsilon}\left(\mathbf{v}_{h}\right)=\frac{1}{2}\left(\left\langle\nabla \mathbf{v}_{h}\right\rangle+\left\langle\nabla \mathbf{v}_{h}^{T}\right\rangle-\nabla \mathbf{v}_{h}-\nabla \mathbf{v}_{h}^{T}\right)$.

It follows after integration that $\int_{K} \bar{\varepsilon}\left(\pi^{K} \mathbf{v}_{h}-\mathbf{v}_{h}\right)=\overline{0}$.

The last point is to check that $\pi^{K}$ is computable for each basis function. It is clear that all terms but $\left\langle\nabla \varphi_{i}^{k}\right\rangle$ do not cause difficulties. This mean gradient can not be directly evaluated since the function $\varphi_{i}^{k}$ is not known inside $K$. Going back to scalar functions thanks to the identity $\nabla \varphi_{i}^{k}=$ $\mathbf{e}_{k} \otimes \nabla \varphi_{i}(k=x, y, z)$, we have to discuss the computation of $\left\langle\nabla \varphi_{i}\right\rangle$ depending on the dimension of the problem.

In two dimensions, we know that the basis functions are first order polynomial on the edges of $K$. Thanks to Stokes formula, we can exactly compute the mean gradient using the degrees of freedom $\left(\varphi_{i}\left(V_{j}\right)=\delta_{i j}\right)$ :

$\frac{1}{|K|} \int_{K} \nabla \varphi_{i}=\frac{1}{|K|} \int_{\partial_{K}} \varphi_{i} \mathbf{n}=\frac{1}{2|K|}\left(\left|e_{i}^{-}\right| \mathbf{n}_{i}^{-}+\left|e_{i}^{+}\right| \mathbf{n}_{i}^{+}\right)$,

where quantities $\left|e_{i}^{-}\right|$and $\mathbf{n}_{i}^{-}$(resp..$^{+}$) refers to the length and the outward unit normal of the edge of $K$ preceding (resp. following) vertex $V_{i}$. This allows to compute the 2D projection $\pi^{K} \varphi_{i}^{k}$ by

$\pi^{K} \varphi_{i}^{k}=\frac{1}{M_{K}} \mathbf{e}_{k}+\left(\mathbf{e}_{k} \otimes \frac{1}{2|K|}\left(\left|e_{i}^{-}\right| \mathbf{n}_{i}^{-}+\left|e_{i}^{+}\right| \mathbf{n}_{i}^{+}\right)\right)\left(\mathbf{x}-\overline{\mathbf{x}_{K}}\right)$.

In three dimensions, Stokes's formula implies

$\frac{1}{|K|} \int_{K} \nabla \varphi_{i}=\frac{1}{|K|} \sum_{f} \int_{f} \varphi_{i} \mathbf{n}_{f}$

where $\mathbf{n}_{f}$ is the unit outward normal to the face $f$. This requires the computation of $\int_{f} \varphi_{i}$ on the faces of $K$. At this stage, the choice made for $V_{h, s c a l}^{f}$ on the polygonal faces in the definition of the 3D approximation space $V_{h, s c a l}^{K}$ (13) becomes clear: the additional property $\int_{f} \pi^{f, 0} v_{h}=\int_{f} v_{h}$ satisfied for $v_{h} \in V_{h, \text { scal }}^{f}$ allows us to replace the computation of $\int_{f} \varphi_{i}$ by $\int_{f} \pi^{f, 0} \varphi_{i}$. This is a good point since the first integral is not computable using only the degrees of freedom, whereas the last one is computable. Even more, the expression of the $L^{2}$ projection $\pi^{f, 0}$ matches the definition (21) of 
$\pi^{K}$ applied on a face $f$. Consequently, reusing the expression given by (22),

$$
\begin{aligned}
\int_{f} \varphi_{i} & =\int_{f} \pi^{f, 0} \varphi_{i} \\
& =\int_{f}\left(\frac{1}{M_{f}}+\frac{1}{2|f|}\left(\left|e_{i}^{-}\right| \mathbf{n}_{i}^{-}+\left|e_{i}^{+}\right| \mathbf{n}_{i}^{+}\right) \cdot\left(\mathbf{x}-\overline{\mathbf{x}_{f}}\right)\right)
\end{aligned}
$$

where $M_{f}$ is the number of nodes of $f$ and $\overline{\mathbf{x}_{f}}$ is the vertex averaged centroid of $f$. Now dealing with a quantity belonging to $\mathbb{P}_{1}$, the last integral is exactly computed by taking its value at $\mathbf{x}^{f}$, the barycenter of $f$. Denoting by $F_{i, K}$ is the set of faces of $K$ sharing node $i$,

$$
\begin{aligned}
& \frac{1}{|K|} \int_{K} \nabla \varphi_{i}= \\
& \frac{1}{|K|} \sum_{f \in F_{i, K}}\left(\frac{|f|}{M_{f}}+\frac{1}{2}\left(\left|e_{i}^{-}\right| \mathbf{n}_{i}^{-}+\left|e_{i}^{+}\right| \mathbf{n}_{i}^{+}\right) \cdot\left(\mathbf{x}^{f}-\overline{\mathbf{x}_{f}}\right)\right) \mathbf{n}_{f} .
\end{aligned}
$$

Finally, this expression of the mean gradient is injected into (21) to compute the $3 \mathrm{D}$ projection

$$
\begin{aligned}
& \pi^{K} \varphi_{i}^{k}=\frac{1}{M_{K}} \mathbf{e}_{k}+\left(\mathbf{x}-\overline{\mathbf{x}_{K}}\right) \cdot\left(\mathbf{e}_{k} \otimes \frac{1}{|K|}\right. \\
&\left.\sum_{f \in F_{i, K}}\left(\frac{|f|}{M_{f}}+\frac{1}{2}\left(\left|e_{i}^{-}\right| \mathbf{n}_{i}^{-}+\left|e_{i}^{+}\right| \mathbf{n}_{i}^{+}\right) \cdot\left(\mathbf{x}^{f}-\overline{\mathbf{x}_{f}}\right)\right) \mathbf{n}_{f}\right) .
\end{aligned}
$$

In both cases, because the projection $\pi^{K}\left(\varphi_{i}^{k}\right)$ is computable from degrees of freedom for each basis function $\varphi_{i}^{k}$, the local stiffness matrices can be computed using (18) in order to assemble the global matrix. We do not give more details on this part and refer to [2].

\subsection{TPFA for the fluid equation}

In this part, we now focus on the fluid equation (9) in which we omit the coupling term $\alpha \operatorname{div}(\mathbf{u})$ :

$$
\begin{aligned}
\forall K \in \tau_{h}, \int_{K} c_{0} p_{h}^{n}+\Delta t \sum_{\substack{f \in \partial K \\
f \notin \Gamma_{N_{p}}}} \mathscr{F}_{K f}^{n} \\
\quad=\int_{K}\left(\Delta t q^{n}+c_{0} p_{h}^{n-1}\right)-\Delta t \int_{K \cap \Gamma_{N_{p}}} \phi_{N}^{n}
\end{aligned}
$$

and we discuss the choices made for the space $Q_{h}$ and for the numerical approximation of the flux $\mathscr{F}_{K f}^{n}$. Among the several existing "finite volume" approximations (see [17] for a modern review), we restrict ourself to the ones using cell centered unknowns. This choice of unknowns location for the discrete pressure will make the evaluation of coupling terms in the next subsection easier. Consequently, $Q_{h}$ is the space of functions that are constant on each cell:

$Q_{h}=\left\{p_{h} \in L^{2}(\Omega): p_{h_{\mid K}} \in \mathbb{P}_{0}(K)\right\}$,

which implies that $Q_{h}$ is not a subset of $Q$. The family of functions $\left\{\phi_{K}\right\}_{K \in \tau_{h}}$ where $\phi_{K}(\mathbf{x})$ equals 1 if $\mathbf{x}$ is inside the cell $K$ and 0 otherwise is a basis for the space $Q_{h}$. Any element of $Q_{h}$ can thus be expanded over this basis by writing $p_{h}=\sum_{K \in \tau_{h}} p_{h_{\mid K}} \phi_{K}$.

The simplest numerical approximation for the flux $\mathscr{F}_{K f}^{n}$ is called Two Point Flux Approximation. This approximation has a simple formulation, using only information from neighboring cells, meaning a low stencil and thus a low computational cost. It also satisfies interesting properties such as accuracy, stability or maximum principle. Unfortunately, it requires the mesh to satisfy the $\bar{\kappa}$-orthogonality condition: for each face $f$ connecting a cell $K$ of computational center $\mathbf{x}_{K}$ (which possibly differs from the barycenter $\mathbf{x}^{K}$ ) with a cell $L$ of computational center $\mathbf{x}_{L}$, the scheme is consistent only if

$\bar{\kappa}^{-1}\left(\mathbf{x}_{K} \mathbf{x}_{L}\right) \perp f$.

Building such meshes is a challenge, especially when the tensor $\bar{\kappa}$ is anisotropic. However, this flux approximation is still a good choice to start with. For now, assume that $\bar{\kappa}=$ $\kappa \overline{I_{d}}$ where $\kappa \in \mathbb{R}$ may differ from cell to cell. The harmonic mean permeability between cells $K$ and $L$ is defined as

$\kappa_{K L}=\left(d_{K f}+d_{L f}\right)\left(\frac{d_{L f}}{\kappa_{L}}+\frac{d_{K f}}{\kappa_{K}}\right)^{-1}$,

where $d_{K f}$ (resp. L) is the distance between cell center $\mathbf{x}_{K}$ (resp. L) and face $f$. The numerical flux is then defined from the transmissivity term $T_{f}$ by

Finite Volume: Two Point Flux Approxi-
mation
$\mathscr{F}_{K f}^{n}= \begin{cases}T_{f}\left(p_{h_{\mid K}}^{n}-p_{h_{\mid L}}^{n}\right) & \text { with } T_{f}=\frac{|f| \kappa_{K L}}{d_{K L}} \text { if } f \subset \Omega \\ T_{f} p_{h_{\mid K}}^{n} & \text { with } T_{f}=\frac{|f| \kappa_{K}}{d_{K f}} \text { if } f \subset \Gamma_{D_{p}} .\end{cases}$

and used to define the discrete $H^{1}$ seminorm

$$
\begin{array}{r}
\left|p_{h}\right|_{1, \tau}=\left(\sum_{f \subset \bar{\Omega} \backslash \Gamma_{N_{p}}} T_{f} D_{f}\left(p_{h}\right)^{2}\right)^{\frac{1}{2}} \\
\text { with } D_{f}\left(p_{h}\right)= \begin{cases}\mid p_{h_{\mid K}}-p_{h_{\mid L} \mid} & \text { if } f \subset \Omega, \\
\left|p_{h_{\mid K}}\right| & \text { if } f \subset \Gamma_{D_{p}} .\end{cases}
\end{array}
$$

If the coefficients $p_{h_{k}}$ are stored in a vector $\mathscr{P}$ and the flux is defined by (26), the equation (24) has the matrix 
form $\mathscr{F} \mathscr{P}=\mathscr{L}^{p}$. The coefficients of the matrix $\mathscr{F}$ of size $\operatorname{card}\left(\tau_{h}\right) \times \operatorname{card}\left(\tau_{h}\right)$ are

$\mathscr{F}_{K K}=|K|+\Delta t \sum_{L \in v(K)} T_{f} \quad$ and $\quad \mathscr{F}_{K L}=-\Delta t T_{f}$

where $v(K)$ is the set of neighboring cells of $K$ and $T_{f}$ is the transmissivity between cells $K$ and $L$. The right-hand side $\mathscr{L}^{p}$ includes the discretization of the source term $q$, the contribution of Neumann's boundary conditions and the backward pressure $p^{n-1}$ coming from the time discretization:

$\mathscr{L}_{K}^{p}=c_{0}|K| p_{h_{\mid K}}^{n-1}+\Delta t\left(\int_{K} q^{n}-\int_{K \cap \Gamma_{N_{p}}} \phi_{N}^{n}\right)$.

The two last integrals can be computed from the prescribed functions using low order quadratures rules.

\subsection{The specific coupled scheme}

The definitions of discrete spaces and operators introduced in the two preceding subsections can now be used to make precise the generic coupled scheme (10). Keeping the same basis expansion of $\mathbf{u}_{h}$ and $p_{h}$ and building the concatenated vector of unknowns $[\mathscr{U}, \mathscr{P}]$, the scheme rewrites in matrix form

$\left[\begin{array}{cc}\mathscr{A}_{h} & -\mathscr{B} \\ \mathscr{B}^{T} & \mathscr{F}\end{array}\right]\left[\begin{array}{c}\mathscr{U} \\ \mathscr{P}\end{array}\right]=\left[\begin{array}{c}\mathscr{L}^{u} \\ \mathscr{L}^{p}+\mathscr{L}^{c}\end{array}\right]$

where the expressions of $\mathscr{A}_{h}, \mathscr{F}, \mathscr{L}^{u}$ and $\mathscr{L}^{p}$ have been detailed above. whereas $\mathscr{B}$ and $\mathscr{L}^{c}$ are new quantities due to the coupling terms. Indeed, this coupled formulation involves a new rectangular matrix $\mathscr{B}$ of size $\operatorname{card}\left(\mathscr{M}^{\text {int }}\right) \times$ $\operatorname{card}\left(\tau_{h}\right)$ whose coefficients are computable:

$$
\begin{aligned}
\mathscr{B}\left(\varphi_{j}^{l}, \phi_{k}\right) & =\alpha \int_{\Omega} \phi_{k} \operatorname{div}\left(\varphi_{j}^{l}\right) \\
& =\alpha \int_{K} \operatorname{div}\left(\varphi_{j}^{l}\right) \text { since } \phi_{K}=0 \text { outside } \mathrm{K} \\
& =\alpha \int_{K} \nabla \varphi_{j} \cdot \mathbf{e}_{l} \text { because } \varphi_{j}^{l}=\varphi_{j} \mathbf{e}_{l} .
\end{aligned}
$$

This integral reduces to the computation of $\left\langle\nabla \varphi_{j}\right\rangle$ in the cell $K$, which has already been done in the virtual element method to assemble the stiffness matrix. From this perspective, the use of cell centered unknowns for pressure avoids additional computations when assembling the coupling matrix.

The coupled scheme also modifies the right-hand side of the fluid equation, by taking into account the divergence of the displacement at the previous time step coming from the implicit time discretization:

$\mathscr{L}_{K}^{c}=\alpha \int_{K} \operatorname{div}\left(\mathbf{u}_{h}^{n-1}\right)=\alpha|K| \sum_{i \in \mathscr{M}_{K}}\left\langle\nabla \varphi_{i}\right\rangle^{K} \cdot\left(\begin{array}{c}u_{i}^{x} \\ u_{i}^{y} \\ u_{i}^{z}\end{array}\right)^{n-1}$.
Once again, computing the divergence of the basis functions is equivalent to computing the mean gradient which is already computed. Algorithm 1 sums up the implementation of the coupled scheme. If the mesh remains the same over the different time steps, the computation of the transmissivities and of mean gradients can be done only once. The pres-

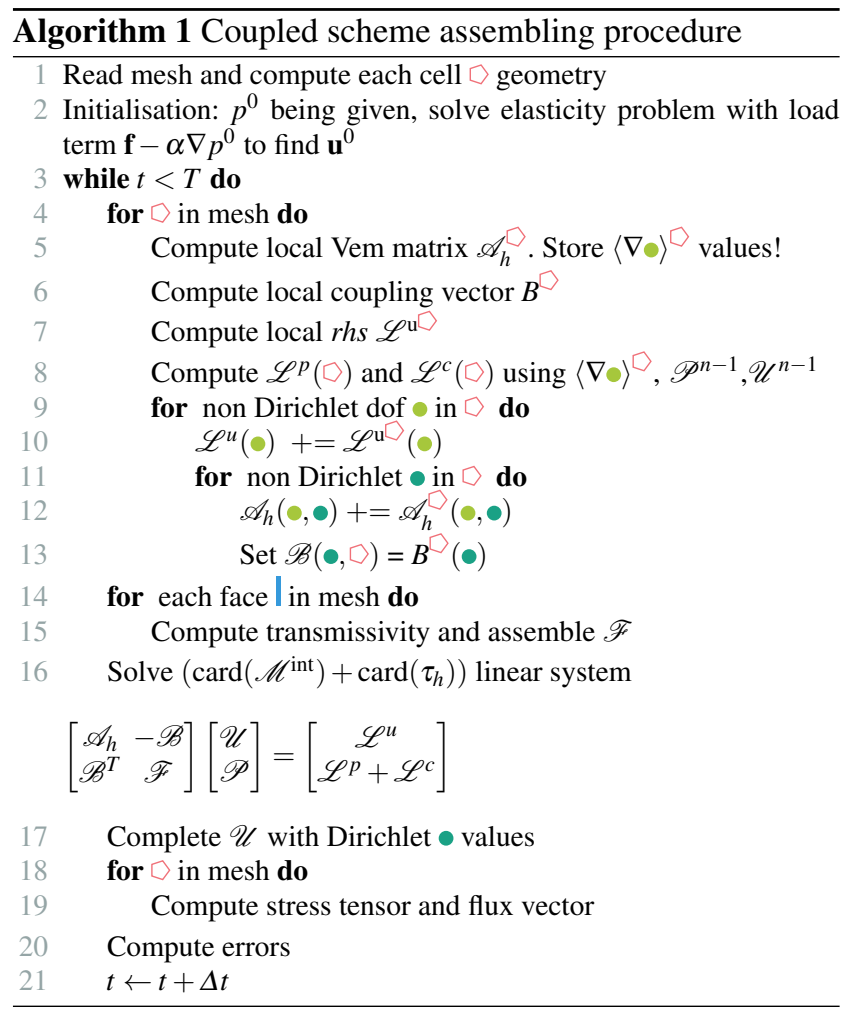

sure gradient (the flux) and the stress tensor are computed using the solution of the linear system. The Darcy velocity is an interpolation of the numerical fluxes computed on each face by (26) since the pressure is known: if $\mathbf{x}^{f}$ and $\mathbf{x}^{K}$ are the barycenter of face $f$ and cell $K$,

$-\kappa \nabla p_{h}\left(\mathbf{x}^{K}\right):=\frac{1}{|K|} \sum_{f \subset \partial K} \mathscr{F}_{K f}^{n}\left(\mathbf{x}^{f}-\mathbf{x}^{K}\right)$.

Since the displacement's basis functions are not known inside the element, the stress tensor can not be computed by directly differentiating the basis functions. Instead, a mean strain tensor is computed in each cell by using again the projection $\pi^{K}$ and is then multiplied by $\overline{\bar{C}}$ to obtain a mean stress tensor in the cell: $\bar{\sigma}_{\pi^{K}\left(\mathbf{u}_{h}\right)}=\overline{\bar{C}} \bar{\varepsilon}\left(\pi^{K} \mathbf{u}_{h}\right)$.

\section{Numerical analysis of the coupled scheme}

This section is devoted to the mathematical analysis of the coupled scheme (10) with the two point flux approximation 
for the finite volume scheme. In order to simplify the developments, the Neumann's boundary conditions are assumed to be zero $\left(\mathbf{t}_{N}=\mathbf{0}, \phi_{N}=0\right)$. Two results are established in this section. The first one is the existence and uniqueness of a discrete solution, and the second one is an error estimate for this discrete solution.

Proposition 2 Scheme (10) using two points approximation for the fluxes (26) admits at each time $t^{n}$ a unique discrete solution $\left(\mathbf{u}_{h}^{n}, p_{h}^{n}\right)$.

Proof Let $\mathscr{O}$ be the operator of system (10). At each time $t^{n}$, we are looking for a pair satisfying $\mathscr{O}\left(u_{h}^{n}, p_{h}^{n}\right)=b$ where $b$ depends on the data and the solution at the previous time step. Considering that

- the operator $\mathscr{O}$ is linear (thanks to the bilinearity of $a_{h}$ and the linearity of the fluxes $\mathscr{F}_{K f}^{n}$ );

- the number of equations ( $d$ for each node plus one for each cell) equals the number of unknowns (see the definition of the discrete spaces);

- at the first time step, $\left(\mathbf{u}_{h}^{0}, p_{h}^{0}\right)$ is uniquely defined by the initial data by setting $p_{h}^{0}$ equals to the projection of $p(t=$ $0, \mathbf{x})$ in the discrete space, and computing $\mathbf{u}_{h}^{0}$ solution of the discrete elasticity problem with $p_{h}=p_{h}^{0}$, which has a unique solution according to lemma 1 ;

it suffices to show that operator $\mathscr{O}$ is invertible, or equivalently that the only solution to the homogeneous system at each time $t^{n}$

$$
\begin{array}{r}
\forall \mathbf{v}_{\mathbf{h}} \in V_{h, 0}, \quad a_{h}\left(\mathbf{u}_{h}^{n}, \mathbf{v}_{h}\right)-\sum_{K} \alpha \int_{K} \operatorname{div}\left(\mathbf{v}_{h}\right) p_{h}^{n}=0 \\
\forall K \subset \bar{\Omega}, \quad \int_{K}\left(c_{0} p_{h}^{n}+\alpha \operatorname{div}\left(\mathbf{u}_{h}^{n}\right)\right)+\Delta t \sum_{\substack{f \subset \partial K \\
f \not \subset \Gamma_{N_{p}}}} \mathscr{F}_{K f}^{n}=0
\end{array}
$$

is $(\mathbf{0}, 0)$. Since $\mathbf{u}_{h}^{n}$ belongs to $V_{h, 0}$, the first equality is valid with $\mathbf{v}_{h}=\mathbf{u}_{h}^{n}$. The second equality is multiplied by the constant value $p_{h_{\mid K}}^{n}$ and then summed over the cells $K$ to write

$$
\begin{aligned}
& a_{h}\left(\mathbf{u}_{h}^{n}, \mathbf{u}_{h}^{n}\right)-\sum_{K} \alpha \int_{K} \operatorname{div}\left(\mathbf{u}_{h}^{n}\right) p_{h}^{n}=0 \\
& \sum_{K} \int_{K}\left(c_{0} p_{h}^{n} p_{h_{\mid K}}^{n}+\alpha \operatorname{div}\left(\mathbf{u}_{h}^{n}\right) p_{h_{\mid K}}^{n}\right)+\Delta t \sum_{K} p_{h_{\mid K}}^{n} \sum_{\substack{f \subset \partial K \\
f \not \subset \Gamma_{N_{p}}}} \mathscr{F}_{K f}^{n}=0 .
\end{aligned}
$$

The terms $\int_{K} \alpha \operatorname{div}\left(\mathbf{u}_{h}^{n}\right) p_{h}^{n}$ cancel upon summing these two equalities:

$$
a_{h}\left(\mathbf{u}_{h}^{n}, \mathbf{u}_{h}^{n}\right)+\int_{\Omega} c_{0} p_{h}^{n} p_{h}^{n}+\Delta t \sum_{K} p_{h_{\mid K}}^{n} \sum_{\substack{f \subset \partial K \\ f \subset \subset \Gamma_{N_{p}}}} \mathscr{F}_{K f}^{n}=0 .
$$

Since $a_{h}$ and the $L^{2}$ scalar product are positive-definite, the two first terms of (30) are non negative. The last sum can be reordered using the flux definition

$$
\begin{aligned}
& \sum_{K} p_{h_{\mid K}}^{n} \sum_{\substack{f \subset \partial K \\
f \not \subset \Gamma_{N_{p}}}} \mathscr{F}_{K f}^{n}=\sum_{f \subset \Gamma_{D_{p}}} T_{f}\left(p_{h_{\mid K}}^{n}\right)^{2} \\
& +\sum_{f \subset \bar{\Omega} \backslash \partial \Omega} T_{f}\left(\left(p_{h_{\mid K}}^{n}-p_{h_{\mid L}}^{n}\right) p_{h_{\mid K}}^{n}+\left(p_{h_{\mid L}}^{n}-p_{h_{\mid K}}^{n}\right) p_{h_{\mid L}}^{n}\right) \\
& =\sum_{f \subset \bar{\Omega} \backslash \partial \Omega} T_{f}\left(p_{h_{\mid K}}^{n}-p_{h_{\mid L}}^{n}\right)^{2}+\sum_{f \subset \Gamma_{D_{p}}} T_{f}\left(p_{h_{\mid K}}^{n}\right)^{2}
\end{aligned}
$$

and is thus also non negative. Because the sum (30) equals zero, each of the three non negative terms must be null. Using again the fact that $a_{h}$ is positive-definite, $a_{h}\left(\mathbf{u}_{h}^{n}, \mathbf{u}_{h}^{n}\right)=0$ implies $\mathbf{u}_{h}^{n}=\mathbf{0}$. The same argument can be used with the $L^{2}$ scalar product if $c_{0}>0$ to show that $p_{h}^{n}=0$. If not, the second part of sum (31) implies that $p_{h_{\mid K}}^{n}=0$ when $f \not \subset \Gamma_{D_{p}}$. Working from this point and proceeding from neighbour to neighbour, the first part of the sum can be used to see that $p_{h_{\mid K}}^{n}=0$ in every other cell, and to conclude that $p_{h}^{n}=0$.

We now want to establish a bound on the difference between the discrete solution $\left(\mathbf{u}_{h}, p_{h}\right)$ and the exact solution $(\mathbf{u}, p)$. Let us start by introducing interpolation operators $P: Q \rightarrow Q_{h}$ and $\tilde{P}: V_{0} \rightarrow V_{h, 0}$ from continuous spaces to discrete spaces defined by

$\forall K \in \tau_{h}, P p(\mathbf{x})=p\left(\mathbf{x}_{K}\right) \quad$ if $\mathbf{x} \in K$,
$\forall K \in \tau_{h},(\tilde{P} \mathbf{u})\left(V_{i}\right)=\mathbf{u}\left(V_{i}\right), \quad i=1, \cdots, M_{K}$.

The error between exact and discrete solutions can be split into an interpolation error $\left(E_{\mathbf{u}}^{I}=\mathbf{u}-\tilde{P} \mathbf{u}\right.$ and $\left.E_{p}^{I}=p-P p\right)$ related to the quality of approximations spaces and an approximation error $\left(E_{\mathbf{u}}^{A}=\tilde{P} \mathbf{u}-\mathbf{u}_{h}\right.$ and $\left.E_{p}^{A}=P p-p_{h}\right)$ related to the quality of the numerical scheme :

$$
\begin{aligned}
& \mathbf{u}-\mathbf{u}_{h}=\mathbf{u}-\tilde{P} \mathbf{u}+\tilde{P} \mathbf{u}-\mathbf{u}_{h}=E_{\mathbf{u}}^{I}+E_{\mathbf{u}}^{A}=E_{\mathbf{u}} \\
& p-p_{h}=p-P p+P p-p_{h}=E_{p}^{I}+E_{p}^{A}=E_{p} .
\end{aligned}
$$

A projection from $V_{0}$ to the space of piecewise affine functions $\mathbb{P}_{1}(K)$ will also be used. The associated projection error shall be denoted $E_{\mathbf{u}}^{\pi}(\mathbf{x})=\mathbf{u}_{\left.\right|_{K}}(\mathbf{x})-\left(\pi^{K} \mathbf{u}_{\mid K}\right)(\mathbf{x})$ for $\mathbf{x} \in K$. The projection $\pi^{K}$ corresponds to the one defined by (21) provided that $\mathbf{u}$ belongs to $\mathscr{C}^{0}(\bar{\Omega}) \cap H^{1}(\Omega)$. Since this quantity does not belong to $H^{1}(\Omega)$, we introduce the broken $H^{1}$ norm

$$
\|\cdot\|_{H^{1}(\tau)}=\left(\sum_{K \in \tau_{h}}\|\cdot\|_{H^{1}(K)}^{2}\right)^{\frac{1}{2}}
$$

Proposition 3 Assuming the exact solution $(\mathbf{u}, p)$ to be sufficiently smooth and the condition $\Delta t \leq \frac{c_{0}}{2}$, the inequality

$$
\left\|E_{\mathbf{u}}^{A, N}\right\|_{H^{1}}^{2}+\left\|E_{p}^{A, N}\right\|_{L^{2}}^{2}+\sum_{n=1}^{N} \Delta t\left|E_{p}^{A, n}\right|_{1, \tau}^{2} \leq B\left(h^{2}+\Delta t^{2}+E^{I}\right)
$$

holds, where $B$ is a positive constant depending on the physical parameters $c_{0}, \alpha, d,|\Omega|, t^{N}$, the time derivatives of the 
true solution $\partial_{t t} \mathbf{u}$ and $\partial_{t t} p$, and initial approximation error $E_{\mathbf{u}}^{A, 0}$, and where $E^{I}$ collects the interpolation errors.

Proof The proof is decomposed in three parts. The aim of the first part is to obtain an inequality involving the approximation errors in the left-hand side. In the second part, the right hand side is bounded by a quantity independent of the approximation errors. In the last part, a Grönwall lemma is used to conclude.

First part In this part, we work at a given time step $t^{n}$ but the superscript $n$ over discrete unknowns is omitted for more readability. The bilinearity of $a$ and $a_{h}$ is used to introduce some terms in the exact formulation (4) and approximate formulation (10) : $\forall \mathbf{v}_{h} \in V_{h, 0}$,

$$
\begin{aligned}
& \sum_{K \in \tau_{h}} a\left(\mathbf{u}_{\left.\right|_{K}}-\pi^{K} \mathbf{u}_{\left.\right|_{K}}, \mathbf{v}_{h}\right)+\sum_{K \in \tau_{h}} a\left(\pi^{K} \mathbf{u}_{\mid K}, \mathbf{v}_{h}\right) \\
&-\alpha \int_{\Omega} p \operatorname{div}\left(\mathbf{v}_{h}\right)=\int_{\Omega} \mathbf{f} \cdot \mathbf{v}_{h} \\
&-a_{h}\left(\tilde{P} \mathbf{u}-\mathbf{u}_{h}, \mathbf{v}_{h}\right)+a_{h}\left(\tilde{P} \mathbf{u}-\sum_{K \in \tau_{h}} \pi^{K} \mathbf{u}_{\left.\right|_{K}}, \mathbf{v}_{h}\right) \\
&+\sum_{K \in \tau_{h}} a_{h}\left(\pi^{K} \mathbf{u}_{\left.\right|_{K}}, \mathbf{v}_{h}\right)-\alpha \int_{\Omega} p_{h} \operatorname{div}\left(\mathbf{v}_{h}\right)=\int_{\Omega} \mathbf{f}_{h} \cdot \mathbf{v}_{h} .
\end{aligned}
$$

Subtracting these two equalities, using for all $K$ the property $a_{h}\left(\pi^{K} \mathbf{u}_{\left.\right|_{K}}, \mathbf{v}_{h}\right)=a\left(\pi^{K} \mathbf{u}_{\left.\right|_{K}}, \mathbf{v}_{h}\right)$

which is valid because $\pi^{K} \mathbf{u}_{\left.\right|_{K}} \in \mathbb{P}_{1}^{d}(K)$, and choosing $\mathbf{v}_{h}=$ $D^{-} E_{\mathbf{u}}^{A}$ leads to

$$
\begin{gathered}
a_{h}\left(E_{\mathbf{u}}^{A}, D^{-} E_{\mathbf{u}}^{A}\right)-\alpha \int_{\Omega} E_{p} \operatorname{div}\left(D^{-} E_{\mathbf{u}}^{A}\right)=a\left(-E_{\mathbf{u}}^{\pi}, D^{-} E_{\mathbf{u}}^{A}\right) \\
+a_{h}\left(E_{\mathbf{u}}^{\pi}-E_{\mathbf{u}}^{I}, D^{-} E_{\mathbf{u}}^{A}\right)+\int_{\Omega}\left(\mathbf{f}-\mathbf{f}_{h}\right) \cdot D^{-} E_{\mathbf{u}}^{A}
\end{gathered}
$$

The exact and approximate pressure equations are treated as well to insert discrete terms

$$
\begin{gathered}
\int_{K} c_{0}\left(D^{-}-D^{-}+\partial_{t}\right)\left(p\left(t^{n}, \mathbf{x}\right)\right)+\int_{\partial K}-\kappa \nabla p\left(t^{n}, \mathbf{x}\right) \cdot \mathbf{n} \\
+\int_{K} \alpha \operatorname{div}\left(\left(D^{-}-D^{-}+\partial_{t}\right)\left(\mathbf{u}\left(t^{n}, \mathbf{x}\right)\right)\right)=\int_{K} q\left(t^{n}, \mathbf{x}\right) \\
\int_{K} c_{0} D^{-} p_{h_{K}}+\alpha \operatorname{div}\left(D^{-} \mathbf{u}_{h}\right)+\sum_{f \subset \partial K} \mathscr{F}_{K f}=\int_{K} q\left(t^{n}, \mathbf{x}\right)
\end{gathered}
$$

and then subtracted, multiplied by the cell constant value $E_{p}^{A}$ and summed over the cells $K$ to obtain

$$
\begin{aligned}
& \int_{\Omega} c_{0} D^{-}\left(E_{p}\right) E_{p}^{A}+\alpha \operatorname{div}\left(D^{-} E_{\mathbf{u}}\right) E_{p}^{A}+\sum_{K f \subset \partial K} \sum_{K f}\left(\overline{F_{K f}}-\mathscr{F}_{K f}\right) E_{p}^{A} \\
& =\int_{\Omega} c_{0}\left(D^{-} p-\partial_{t} p\right) E_{p}^{A}+\int_{\Omega} \alpha \operatorname{div}\left(\left(D^{-} \mathbf{u}-\partial_{t} \mathbf{u}\right)\right) E_{p}^{A}
\end{aligned}
$$

The overlined flux $\overline{F_{K f}}$ stands for the exact flux at time $t^{n}$, $\overline{F_{K f}^{n}}=\int_{f \cap \partial K}-\kappa \nabla p\left(t^{n}, \mathbf{x}\right) \cdot \mathbf{n}$. Splitting the difference $\overline{F_{K f}}-$
$\mathscr{F}_{K f}$ with the use of the discrete flux evaluated at the projection of the exact pressure $F_{K f}^{\star}$ (per definition (26) with $P p$ instead of $p_{h}$ ),

$$
\begin{aligned}
\sum_{K} \sum_{f \subset \partial K}\left(\overline{F_{K f}}-\mathscr{F}_{K f}\right) E_{p}^{A} & =\sum_{K} \sum_{f \subset \partial K}\left(\overline{F_{K f}}-F_{K f}^{\star}\right) E_{p}^{A} \\
& +\sum_{K} \sum_{f \subset \partial K}\left(F_{K f}^{\star}-\mathscr{F}_{K f}\right) E_{p}^{A} \\
& =\sum_{K} \sum_{f \subset \partial K}\left(\overline{F_{K f}}-F_{K f}^{\star}\right) E_{p}^{A}+\left|E_{p}^{A}\right|_{1, \tau}^{2}
\end{aligned}
$$

where the second sum has been reordered as done in (31). To finish the first part, (33) and (34) are summed to obtain

$$
\begin{aligned}
a_{h}\left(E_{\mathbf{u}}^{A}, D^{-} E_{\mathbf{u}}^{A}\right)+\int_{\Omega} c_{0} D^{-} & E_{p}^{A} E_{p}^{A}+\left|E_{p}^{A}\right|_{1, \tau}^{2} \\
& =B_{1}+B_{2}+B_{3}+B_{4}+B_{5}+B_{6}
\end{aligned}
$$

with

$$
\begin{aligned}
B_{1} & =-\alpha \int_{\Omega} \operatorname{div}\left(D^{-} E_{\mathbf{u}}^{I}\right) E_{p}^{A}+\alpha \int_{\Omega} \operatorname{div}\left(D^{-} E_{\mathbf{u}}^{A}\right) E_{p}^{I}, \\
B_{2} & =a^{\Omega}\left(-E_{\mathbf{u}}^{\pi}, D^{-} E_{\mathbf{u}}^{A}\right)+a_{h}\left(E_{\mathbf{u}}^{\pi}-E_{\mathbf{u}}^{I}, D^{-} E_{\mathbf{u}}^{A}\right), \\
B_{3} & =\int_{\Omega} c_{0}\left(D^{-} p-\partial_{t} p\right) E_{p}^{A}+\int_{\Omega} \alpha \operatorname{div}\left(D^{-} \mathbf{u}-\partial_{t} \mathbf{u}\right) E_{p}^{A}, \\
B_{4} & =\int_{\Omega}\left(\mathbf{f}-\mathbf{f}_{h}\right) \cdot D^{-} E_{\mathbf{u}}^{A} \\
B_{5} & =-\sum_{K} \sum_{f \subset \partial K}\left(\overline{F_{K f}}-F_{K f}^{\star}\right) E_{p}^{A} \\
B_{6} & =-c_{0} \int_{\Omega} E_{p}^{A} D^{-} E_{p}^{I} .
\end{aligned}
$$

Second part Here we reintroduce the superscript $n$ and we sum the previous equation over $n$ between 1 and $N$. Using the fact that

$$
\begin{aligned}
& \int_{\Omega} c_{0} D^{-} E_{p}^{A, n} E_{p}^{A, n} \geq \frac{c_{0}}{2 \Delta t}\left(\left\|E_{p}^{A, n}\right\|_{L^{2}}^{2}-\left\|E_{p}^{A, n-1}\right\|_{L^{2}}^{2}\right) \\
& a_{h}\left(E_{\mathbf{u}}^{A, n}, D^{-} E_{\mathbf{u}}^{A, n}\right) \geq \frac{1}{2 \Delta t}\left(\left\|E_{\mathbf{u}}^{A, n}\right\|_{a_{h}}^{2}-\left\|E_{\mathbf{u}}^{A, n-1}\right\|_{a_{h}}^{2}\right)
\end{aligned}
$$

(where $\|\mathbf{u}\|_{a_{h}}^{2}=a_{h}(\mathbf{u}, \mathbf{u})$ ) thanks to the symmetry of $a_{h}$ and of the $L^{2}$ scalar product, we have

$$
\begin{aligned}
& \tilde{\mu}\left\|E_{\mathbf{u}}^{A, N}\right\|_{H^{1}}^{2}+c_{0}\left\|E_{p}^{A, N}\right\|_{L^{2}}^{2}+2 \sum_{n=0}^{N} \Delta t\left|E_{p}^{A, n}\right|_{1, \tau}^{2} \\
& \quad \leq \tilde{\mu}\left\|E_{\mathbf{u}}^{A, 0}\right\|_{H^{1}}^{2}+2 \Delta t \sum_{n=1}^{N}\left(B_{1}^{n}+B_{2}^{n}+B_{3}^{n}+B_{4}^{n}+B_{5}^{n}+B_{6}^{n}\right),
\end{aligned}
$$

where we also used the coercivity constant of $a_{h}$ denoted $\tilde{\mu}$ and the fact that $E_{p}^{A, 0}=0$. Each component of the right-hand side is going to be bounded using the following tools:

1. Young's inequality $2 a b \leq \varepsilon a^{2}+\frac{b^{2}}{\varepsilon}$ with $\varepsilon>0$. When dealing with a summation over $n$, we often choose a different $\varepsilon$ for the last time step;

2. $\forall \mathbf{v} \in\left(H^{1}(\Omega)\right)^{d},\|\operatorname{div}(\mathbf{v})\|_{L^{2}} \leq \sqrt{d}|\mathbf{v}|_{H^{1}}$; 
3. Taylor expansions

$$
\begin{aligned}
D^{-} v\left(t^{n}\right) & =\frac{1}{\Delta t} \int_{t^{n-1}}^{t^{n}} \partial_{t} v(s) d s \\
D^{-} v\left(t^{n}\right)-\partial_{t} v\left(t^{n}\right) & =\frac{1}{\Delta t} \int_{t^{n-1}}^{t^{n}} \partial_{t t} v(s)\left(t^{n-1}-s\right) d s
\end{aligned}
$$

4. Discrete partial summation

$$
\begin{aligned}
\sum_{k=1}^{N} \mathscr{B}\left(a_{k}, b_{k}-b_{k-1}\right)=- & \sum_{k=1}^{N-1} \mathscr{B}\left(b_{k}, a_{k+1}-a_{k}\right) \\
& +\mathscr{B}\left(a_{N}, b_{N}\right)-\mathscr{B}\left(a_{1}, b_{0}\right)
\end{aligned}
$$

for any symmetric bilinear form $\mathscr{B}$;

5. Double Cauchy-Schwarz identity

$$
\left|\int_{\Omega}\left(b^{n}-b^{n-1}\right) a^{n}\right| \leq\left\|a^{n}\right\|_{L^{2}}\left(\Delta t \int_{t^{n-1}}^{t^{n}}\left\|\partial_{t} b(s)\right\|_{L^{2}}^{2} d s\right)^{\frac{1}{2}}
$$

for any scalar or vectorial function $b(\mathbf{x}, t)$ with $b^{n}:=$ $b\left(\mathbf{x}, t^{n}\right)$.

After some developments (detailed in appendix A), we show that for any $\varepsilon_{r}>0$ and $\varepsilon_{l}>0$, defining $\tilde{\varepsilon}_{r}$ by $\frac{1}{\tilde{\varepsilon}_{r}}=1+\frac{1}{\varepsilon_{r}}$,

$$
\begin{aligned}
& \left|2 \Delta t \sum_{n=1}^{N} B_{1}^{n}\right| \leq \frac{\varepsilon_{r}}{4} \sum_{n=1}^{N-1} \Delta t\left\|E_{p}^{A, n}\right\|_{L^{2}}^{2}+\frac{\varepsilon_{r}}{4} \sum_{n=1}^{N-1} \Delta t\left\|E_{\mathbf{u}}^{A, n}\right\|_{H^{1}}^{2} \\
& +\frac{\Delta t}{4}\left\|E_{p}^{A, N}\right\|_{L^{2}}^{2}+\frac{\varepsilon_{l}}{4}\left\|E_{\mathbf{u}}^{A, N}\right\|_{H^{1}}^{2}+\frac{\varepsilon_{l}}{4}\left\|E_{\mathbf{u}}^{A, 0}\right\|_{H^{1}}^{2} \\
& +\frac{4 d \alpha^{2}}{\tilde{\varepsilon}_{r}}\left\|\partial_{t} E_{\mathbf{u}}^{I}\right\|_{L^{2}\left(0, T ; H^{1}\right)}^{2}+\frac{4 d \alpha^{2}}{\varepsilon_{l}}\left(\left\|E_{p}^{I, N}\right\|_{L^{2}}^{2}+\left\|E_{p}^{I, 1}\right\|_{L^{2}}^{2}\right) \\
& +\frac{4 d \alpha^{2}}{\varepsilon_{r}}\left\|\partial_{t} E_{p}^{I}\right\|_{L^{2}\left(0, T ; L^{2}\right)}^{2}
\end{aligned}
$$

$$
\begin{aligned}
& \left|2 \Delta t \sum_{n=1}^{N} B_{2}^{n}\right| \leq \frac{\varepsilon_{r}}{2} \sum_{n=1}^{N-1} \Delta t\left\|E_{\mathbf{u}}^{A, n}\right\|_{H^{1}}^{2}+\frac{\varepsilon_{l}}{2}\left\|E_{\mathbf{u}}^{A, N}\right\|_{H^{1}}^{2} \\
& +\frac{\varepsilon_{l}}{2}\left\|E_{\mathbf{u}^{A, 0}}^{A,}\right\|_{H^{1}}^{2}+\frac{4 \tilde{M}^{2}}{\varepsilon_{l}}\left(\left\|E_{\mathbf{u}^{I, 1}}^{I}\right\|_{H^{1}}^{2}+\left\|E_{\mathbf{u}}^{I, N}\right\|_{H^{1}}^{2}\right) \\
& +\frac{4 \tilde{M}^{2}}{\varepsilon_{r}}\left\|\partial_{t} E_{\mathbf{u}}^{I}\right\|_{L^{2}\left(0, T ; H^{1}\right)}^{2}+\frac{8}{\varepsilon_{r}}\left(M^{2}+\tilde{M}^{2}\right)\left\|\partial_{t} E_{\mathbf{u}}^{\pi}\right\|_{L^{2}\left(0, T ; H^{1}(\tau)\right)}^{2} \\
& +\frac{8}{\varepsilon_{l}}\left(M^{2}+\tilde{M}^{2}\right)\left(\left\|E_{\mathbf{u}}^{\pi, 1}\right\|_{H^{1}(\tau)}^{2}+\left\|E_{\mathbf{u}}^{\pi, N}\right\|_{H^{1}(\tau)}^{2}\right)
\end{aligned}
$$

$$
\begin{aligned}
\mid 2 \Delta t & \sum_{n=1}^{N} B_{3}^{n} \mid \leq \frac{2 \varepsilon_{r}}{4} \sum_{n=1}^{N-1} \Delta t\left\|E_{p}^{A, n}\right\|_{L^{2}}^{2}+\frac{2 \Delta t}{4}\left\|E_{p}^{A, N}\right\|_{L^{2}}^{2} \\
+ & \frac{4}{3} \Delta t^{2}\left(c_{0}^{2} \frac{\left\|\partial_{t t} p\right\|_{L^{2}\left(0, T ; L^{2}\right)}^{2}}{\tilde{\varepsilon}_{r}}+d \alpha^{2} \frac{\left\|\partial_{t t} \mathbf{u}\right\|_{L^{2}\left(0, T ; H^{1}\right)}^{2}}{\tilde{\varepsilon}_{r}}\right)
\end{aligned}
$$

$$
\begin{aligned}
& \left|2 \Delta t \sum_{n=1}^{N} B_{4}^{n}\right| \leq \frac{\varepsilon_{r}}{4} \sum_{n=1}^{N-1} \Delta t\left\|E_{\mathbf{u}}^{A, n}\right\|_{H^{1}}^{2}+\frac{\varepsilon_{l}}{4}\left\|E_{\mathbf{u}}^{A, N}\right\|_{H^{1}}^{2} \\
& +\frac{4}{\varepsilon_{r}}\left\|\partial_{t} E_{\mathbf{f}}^{I}\right\|_{L^{2}\left(0, T ; L^{2}\right)}^{2}+\frac{4}{\varepsilon_{l}}\left(\left\|E_{\mathbf{f}}^{I, N}\right\|_{L^{2}}^{2}+\left\|E_{\mathbf{f}}^{I, 1}\right\|_{L^{2}}^{2}\right)+\frac{\varepsilon_{l}}{4}\left\|E_{\mathbf{u}}^{A, 0}\right\|_{H^{1}}^{2} \\
& \left|2 \Delta t \sum_{n=1}^{N} B_{5}^{n}\right| \leq \sum_{n=1}^{N} \Delta t\left|E_{p}^{A, n}\right|_{1, \tau}^{2}+\sum_{n=1}^{N} \Delta t \sum_{f \subset \bar{\Omega} \backslash \Gamma_{N_{p}}} \frac{\left|\overline{F_{K f}^{n}}-F_{K f}^{\star, n}\right|^{2}}{T_{f}} \\
& \left|2 \Delta t \sum_{n=1}^{N} B_{6}^{n}\right| \leq \frac{\varepsilon_{r}}{4} \sum_{n=1}^{N-1} \Delta t\left\|E_{p}^{A, n}\right\|_{L^{2}}^{2}+\frac{\Delta t}{4}\left\|E_{p}^{A, N}\right\|_{L^{2}}^{2} \\
& +4 c_{0}^{2} \frac{\left\|\partial_{t} E_{p}^{I}\right\|_{L^{2}\left(0, T ; L^{2}\right)}^{2}}{\tilde{\varepsilon}_{r}}
\end{aligned}
$$

where $M$ and $\tilde{M}$ (resp. $\mu$ and $\tilde{\mu}$ ) depends on the continuity (resp. coercivity) constants of $a$ and $a_{h}$. The two terms $\sum_{f \subset \bar{\Omega} \backslash \Gamma_{N_{p}}} \frac{\left|\overline{F_{K f}^{n}}-F_{K f}^{\star, n}\right|^{2}}{T_{f}}$ and $E_{\mathbf{f}}^{I}=\mathbf{f}-\mathbf{f}_{h}$, are assimilated to interpolation errors because they only depend on the true solution and discretization spaces and are thus left in the right-hand side.

By combining these inequalities,

$$
\begin{aligned}
& \left(\tilde{\mu}-\varepsilon_{l}\right)\left\|E_{\mathbf{u}}^{A, N}\right\|_{H^{1}}^{2}+\left(c_{0}-\Delta t\right)\left\|E_{p}^{A, N}\right\|_{L^{2}}^{2}+\sum_{n=0}^{N} \Delta t\left|E_{p}^{A, n}\right|_{1, \tau}^{2} \\
& \leq \varepsilon_{r} \sum_{n=1}^{N-1} \Delta t\left(\left\|E_{\mathbf{u}}^{A, n}\right\|_{H^{1}}^{2}+\left\|E_{p}^{A, n}\right\|_{L^{2}}^{2}\right)+\tilde{B}\left(h^{2}+\Delta t^{2}+E^{I}\right)
\end{aligned}
$$

with $\tilde{B}$ depending on $d, \alpha, c_{0}, M, \tilde{M}, \mu, \tilde{\mu}, t^{N}, E_{\mathbf{u}}^{A, 0}, \partial_{t t} p, \partial_{t t} \mathbf{u}$, $\varepsilon_{l}$ and $\varepsilon_{r}$. The term $E^{I}$ collects all the interpolation (or assimilated) errors, ie. interpolation error on the unknown $\left\|E_{\mathbf{u}}^{I}\right\|_{L^{\infty}\left(0, T ; H^{1}\right)}^{2}\left\|\partial_{t} E_{\mathbf{u}}^{I}\right\|_{L^{2}\left(0, T ; H^{1}\right)}^{2},\left\|E_{p}^{I}\right\|_{L^{\infty}\left(0, T ; L^{2}\right)}^{2}$ and $\left\|\partial_{t} E_{p}^{I}\right\|_{L^{2}\left(0, T ; L^{2}\right)}^{2}$, projection error $\left\|E_{\mathbf{u}}^{\pi}\right\|_{L^{\infty}\left(0, T ; H^{1}(\tau)\right)}^{2}$ and $\left\|\partial_{t} E_{\mathbf{u}}^{\pi}\right\|_{L^{2}\left(0, T ; H^{1}(\tau)\right)}^{2}$, interpolation error on the load function $\left\|E_{\mathbf{f}}^{I}\right\|_{L^{\infty}\left(0, T ; L^{2}\right)}^{2}$ and $\left\|\partial_{t} E_{\mathbf{f}}^{I}\right\|_{L^{2}\left(0, T ; L^{2}\right)}^{2}$ and finite volume interpolation error $\sum_{n=1}^{N} \Delta t \sum_{f \in \bar{\Omega} \backslash \Gamma_{N_{p}}} T_{f}^{-1}\left|\overline{F_{K f}^{n}}-F_{K f}^{\star, n}\right|^{2}$. Note that in three dimensions, the $\mathscr{O}\left(h^{-1}\right)$ term $T_{f}^{-1}$ cancels with the flux error and therefore is not divergent.

Third part Using now the hypothesis that $\Delta t$ is small enough compared to $c_{0}, \Delta t$ disappears in the left-hand side. We then choose $\varepsilon_{l}<\tilde{\mu}$ and set $\varepsilon_{r}=\min \left\{\tilde{\mu}-\varepsilon_{l}, \frac{c_{0}}{2}\right\}$ to have, after dividing by $\varepsilon_{r}$,

$$
\begin{aligned}
\left\|E_{\mathbf{u}}^{A, N}\right\|_{H^{1}}^{2}+\left\|E_{p}^{A, N}\right\|_{L^{2}}^{2}+\sum_{n=0}^{N} \Delta t\left|E_{p}^{A, n}\right|_{1, \tau}^{2} \\
\quad \leq \sum_{n=0}^{N-1} \Delta t\left(\left\|E_{\mathbf{u}}^{A, n}\right\|_{H^{1}}^{2}+\left\|E_{p}^{A, n}\right\|_{L^{2}}^{2}\right)+B\left(h^{2}+\Delta t^{2}+E^{I}\right)
\end{aligned}
$$

with $B$ depending on $d, \alpha, c_{0}, M, \tilde{M}, \mu, \tilde{\mu}, t^{N}, \partial_{t t} \mathbf{u}, \partial_{t t} p$ and $E_{\mathbf{u}}^{A, 0}$. We also added the positive quantity $\Delta t\left(\left\|E_{\mathbf{u}}^{A, 0}\right\|_{H^{1}}^{2}+\right.$ $\left.\left\|E_{p}^{A, 0}\right\|_{L^{2}}^{2}\right)$ to the right-hand side in order to conclude using a discrete version of Grönwall's lemma: 
Lemma 4 Let $\left(a_{n}\right)_{n \geq 0},\left(b_{n}\right)_{n \geq 0}$ be sequences of nonnegative numbers and $C \in \mathbb{R}^{+}$. Assume that

$$
a_{0}+b_{0} \leq C
$$

and that there exists $\lambda>0$ such that

$$
\forall N \geq 1, \quad a_{N}+b_{N} \leq \lambda \sum_{m=0}^{N-1} a_{m}+C .
$$

Then there holds

$$
\forall N \geq 0, \quad a_{N}+b_{N} \leq C \exp (\lambda N) .
$$

This lemma is applied with $a_{n}=\left\|E_{\mathbf{u}}^{A, n}\right\|_{H^{1}}^{2}+\left\|E_{p}^{A, n}\right\|_{L^{2}}^{2}, \lambda=$ $\Delta t, b_{n}=\sum_{m=0}^{n} \Delta t\left|E_{p}^{A, m}\right|_{1, \tau}^{2}$ and $C=B$. Note that $\exp (N \Delta t)=$ $\exp \left(t^{N}\right)$ is independent of $\Delta t$ and consequently included in $B$.

The next step of the numerical analysis is to bound the interpolation errors. This task invokes classical arguments of polynomial approximation in Sobolev Spaces - see eg [13] and [18] for the finite volume part - not detailed here and leads to the estimate

Theorem 5 Under the hypotheses of proposition 3, there exists $C$ depending on $c_{0}, \alpha, d,|\Omega|, t^{N}, \mathbf{u}, p$ such that

$$
\begin{aligned}
\left\|E_{\mathbf{u}}^{A}\right\|_{L^{\infty}\left(0, T ; H^{1}(\Omega)\right)}^{2}+ & \left\|E_{p}^{A}\right\|_{L^{\infty}\left(0, T ; L^{2}(\Omega)\right)}^{2}+ \\
& \left|E_{p}^{A}\right|_{L^{2}\left(0, T ; H^{1}(\tau)\right)}^{2} \leq C\left(h^{2}+\Delta t^{2}\right) .
\end{aligned}
$$

\section{Numerical illustration}

Two illustrations are proposed in this section. The first one concerns the error estimate established in the previous section. We show that on a simple $2 \mathrm{D}$ test case, we recover the correct order of convergence. The second one performs some computations on a $3 \mathrm{D}$ realistic case.

\subsection{Convergence of the scheme}

We propose here an illustration of theorem 5 on an academic test case taken from [21]. The unit square is filled with a homogeneous medium with elastic coefficients $E=2.5, v=$ 0.25 and a mobility matrix equals to one: $\bar{\kappa}=\kappa \overline{I_{2}}$ with $\kappa=$ 1. The poroelastic parameters are chosen to be $\alpha=1$ and $c_{0}=0.5$ and the simulation time runs from 0 to $T=1$. We consider the analytic solution

$$
\begin{aligned}
& \mathbf{u}(\mathbf{x}, t)=10^{-2} \mathrm{e}^{-t}\left(\begin{array}{c}
x^{2} y \\
-x y^{2}
\end{array}\right), \\
& p(\mathbf{x}, t)=\mathrm{e}^{-t} \sin \left(\frac{x}{\sqrt{2}}\right) \sin \left(\frac{y}{\sqrt{2}}\right),
\end{aligned}
$$

and use it to compute the load terms

$\mathbf{f}(\mathbf{x}, t)=\left(\begin{array}{l}\frac{\alpha \mathrm{e}^{-t}}{\sqrt{2}} \cos (x / \sqrt{2}) \sin (y / \sqrt{2})-2 \cdot 10^{-2} \mathrm{e}^{-t} y \\ \frac{\alpha \mathrm{e}^{-t}}{\sqrt{2}} \sin (x / \sqrt{2}) \cos (y / \sqrt{2})+2.10^{-2} \mathrm{e}^{-t} x\end{array}\right)$,

$q(\mathbf{x}, t)=\left(\kappa-c_{0}\right) \mathrm{e}^{-t} \sin \left(\frac{x}{\sqrt{2}}\right) \sin \left(\frac{y}{\sqrt{2}}\right)$.

Dirichlet conditions are imposed to both unknowns on the boundaries. The domain $\Omega$ is meshed with Vononoï cells (see figure 2): this kind of triangulation satisfies by definition the orthogonality condition (25). Several meshes are generated with a decreasing maximal diameter $h$. Simultaneously, different values are successively taken for the time step: $\Delta t=0.2,0.1,0.02,0.01$. For each pair $(h, \Delta t)$, the approximation error $E^{A}$ defined by equation (35) is computed. This error is plotted on figure 3 as a function of the mesh size $h$ for a given time step (top), or as a function of the time step $\Delta t$ for a given mesh size (bottom). We highlight two asymptotic behaviours. From one side, if the time step is too large, reducing the mesh size does not have any effect on the error: this can be symmetrically seen on the red curve on the left graph or in the upper right area of the right graph. In this case, the $h$ term is negligible compared to the $\Delta t$ term in the error estimation. On the other side, if the mesh size is too coarse, reducing the time step does not improve on the error: this can be symmetrically seen on the upper right corner of the left graph or on the red curve on the right graph. In this case, the $\Delta t$ term is negligible in front of the $h$ term in the error estimation. On both graphs, we can retrieve the correct order of accuracy by looking at the yellow curve, where one variable is small enough not to slow down the convergence when reducing the size of the other. The slope of these yellow curves equals one, which was expected by the error estimate formula. Between the red and the yellow curves lies the area where reducing either the time step or the mesh size can reduce the error.

This double dependence is also represented on figure 4 where we have plotted the contour lines of the approximation error in the $(h, \Delta t)$ plane. Once again, one can see the regions where the error is mostly due to $\Delta t$ (horizontal lines) and the regions where the error is mostly due to $h$ (vertical lines).

\subsection{A realistic subsidence case}

In this part, we illustrate the ability of our numerical scheme to solve a realistic three dimensional case. The aim of this numerical experiment is to simulate the production of an initially overpressured fluid trapped in a stratified underground

The production through a well is here modeled by a pressure boundary condition. The model is made of five horizontal layers of length $15 \mathrm{~km} \times 15 \mathrm{~km}$ whose characteristics 

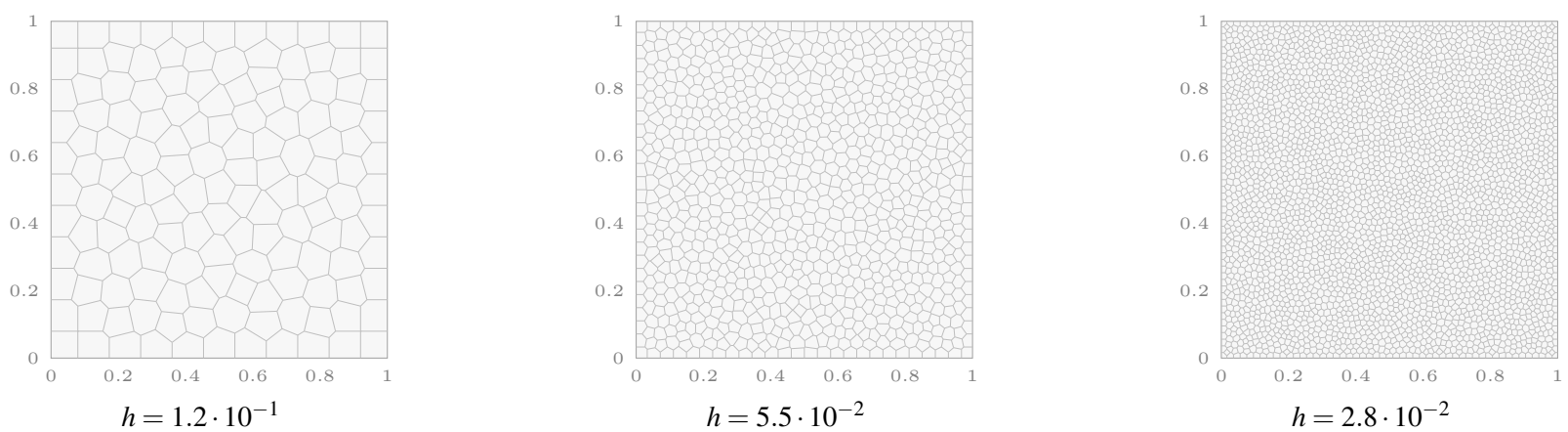

Fig. 2 Voronoï tesselations of the unit square generated with Neper software, see [23]
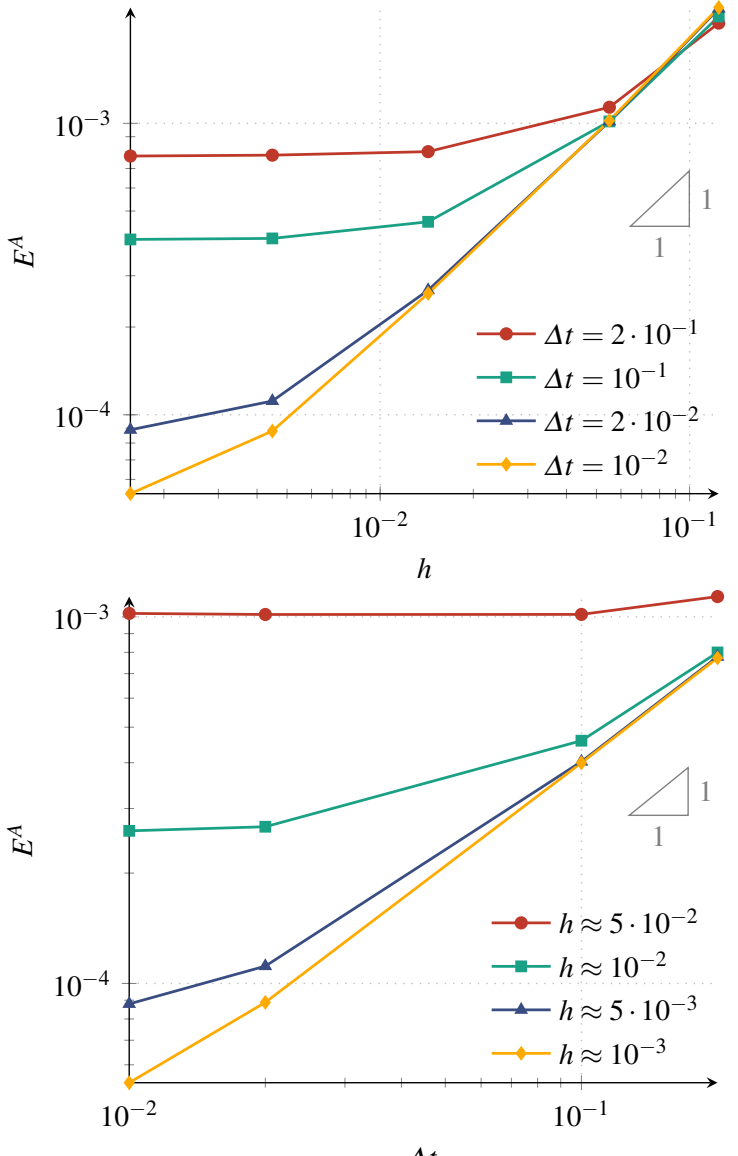

$\Delta t$

Fig. 3 Evolution of the approximation error as a function of mesh size (top) or as a function of time step (bottom)

are detailed in table 1 . The permeability can vary strongly depending of the modelled rock leading to different mobility magnitudes. Here the reservoir layer $(\mathrm{Re})$ is between two low permeability shale layers (Sh). The base and the top of the stratified media is made of two sandstone layers (Ss). The coefficient $c_{0}$ is set from the relation $c_{0}=\phi c_{f}$ where $\phi$ is the porosity of the different layers and $c_{f}=4.5 \cdot 10^{-10}$ $\mathrm{Pa}^{-1}$ is the compressibility of water. Because the reservoir undergoes the weight of the surrounding layers, compaction

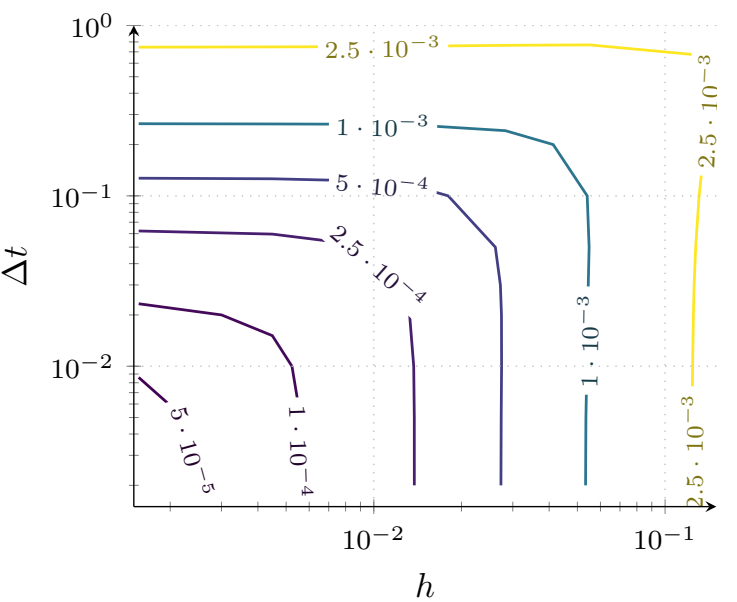

Fig. 4 Approximation error in the $(h, \Delta t)$ plane. Both discretizations can be refined to reduce the error until one of the two parameters becomes negligible compared to the other.

occurs, leading to subsidence. This phenomenon can be associated with plastic strain. Nevertheless, since our model is restricted to linear elasticity in the present study, we model these effects by reducing the Young modulus. We take $E=1$ $\mathrm{GPa}$ inside the reservoir and $E=5 \mathrm{GPa}$ in all the other materials. For the sake of simplicity, the coefficients $v=0.3$ and $\alpha=1$ are taken constant.

The domain is meshed with a $2 \mathrm{D}$ Voronoï discretization which is extruded in the $\mathbf{e}_{z}$ direction as shown on figure 5. The mesh is horizontally refined around the productive part of the well region (the bottom left corner) and is vertically refined around the reservoir layer.

Table 1 Characteristics of the five horizontal layers

\begin{tabular}{rrrrrr}
\hline Layer name & Ss & Sh & Re & Sh & Ss \\
\hline Top altitude (m) & 0 & -2000 & -2100 & -2300 & -2400 \\
Thickness (m) & 2000 & 100 & 200 & 100 & 1600 \\
Mesh layers (-) & 16 & 4 & 8 & 4 & 14 \\
$\kappa\left(\mathrm{m}^{2} \mathrm{~Pa}^{-1} \mathrm{~s}^{-1}\right)$ & $10^{-9}$ & $10^{-15}$ & $10^{-9}$ & $10^{-15}$ & $10^{-9}$ \\
Porosity $\phi(-)$ & 0.2 & 0.1 & 0.3 & 0.1 & 0.2 \\
Initial $\tilde{p}$ (bar) & 0 & 0 & 100 & 100 & 100 \\
\hline
\end{tabular}




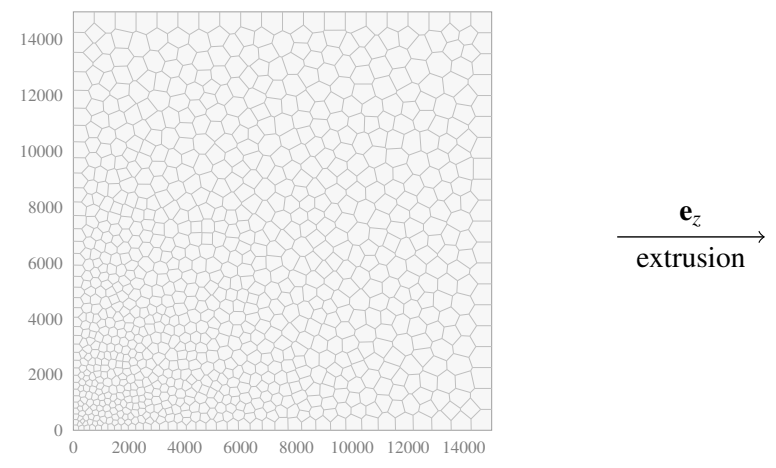

Fig. $52 d$ (left) and 3d (right) view of the extruded mesh

The overpressure reads $\tilde{p}=p-\left(P_{0}-\rho_{f}|\mathbf{g}| z\right)$ where $\rho_{f}=$ $1000 \mathrm{~kg} \cdot \mathrm{m}^{-3}$ is the fluid density and $P_{0}=1$ bar is the atmospheric pressure. At the initial state, the prescribed overpressure depends only on the depth and is also detailed in table 1: it is zero above the reservoir layer and 100 bar below. The elastic equation is used to compute the initial displacement, which is zero in $\mathbf{e}_{x}$ and $\mathbf{e}_{y}$ directions and depends only on $z$ in $\mathbf{e}_{z}$ direction since the only considered mechanical loading comes from the gravity. Once the unknowns are initialized, the simulation covers one year with 20 time steps using a BiCGStab solver preconditioned with a ILU0 method. Although this choice may not be optimal for this coupled and heterogeneous system, it was enough to solve this particular case. The boundary conditions are ( $\mathbf{n}$ being the outward unit normal):

- zero orthogonal displacement $(\mathbf{u} \cdot \mathbf{n})$ and zero flux $(\nabla p$. n) on lateral and bottom faces;

- mechanically free boundary $(\overline{\boldsymbol{\sigma}} \mathbf{n}=\mathbf{0})$ and zero overpressure $(\tilde{p}=0)$ on top face;

- zero overpressure in the corner of the reservoir corresponding to the productive part of the well. In practice, we impose $\tilde{p}=0$ on the lateral faces belonging to the reservoir and sharing a vertex of coordinates $(x=0, y=$ $0)$. This condition simulates an infinite hydraulic connectivity with the surface and triggers the natural extraction of the fluid.

As soon as the zero overpressure condition is imposed on the corner of the reservoir, the fluid starts to move to this point. The reservoir is progressively drained as time goes by. This is shown on figure 6 where the fluid velocity and the pressure are represented in the $(x, y)$ plane inside the reservoir at the end of the simulation. The overpressure tends to zero near the well and is still equal to its initial value (100 bar) at the opposite corner. Because of the permeability barriers constituted by the shale layers, the pressure remains almost constant outside the reservoir. As a consequence of this overpressure drop, the effective stress $\bar{\sigma}^{e}=\bar{\sigma}+\alpha p \bar{I}_{3}$ decreases in the reservoir. This drop is highly visible near the well but tends to vanish away from it, where the overpressure has not
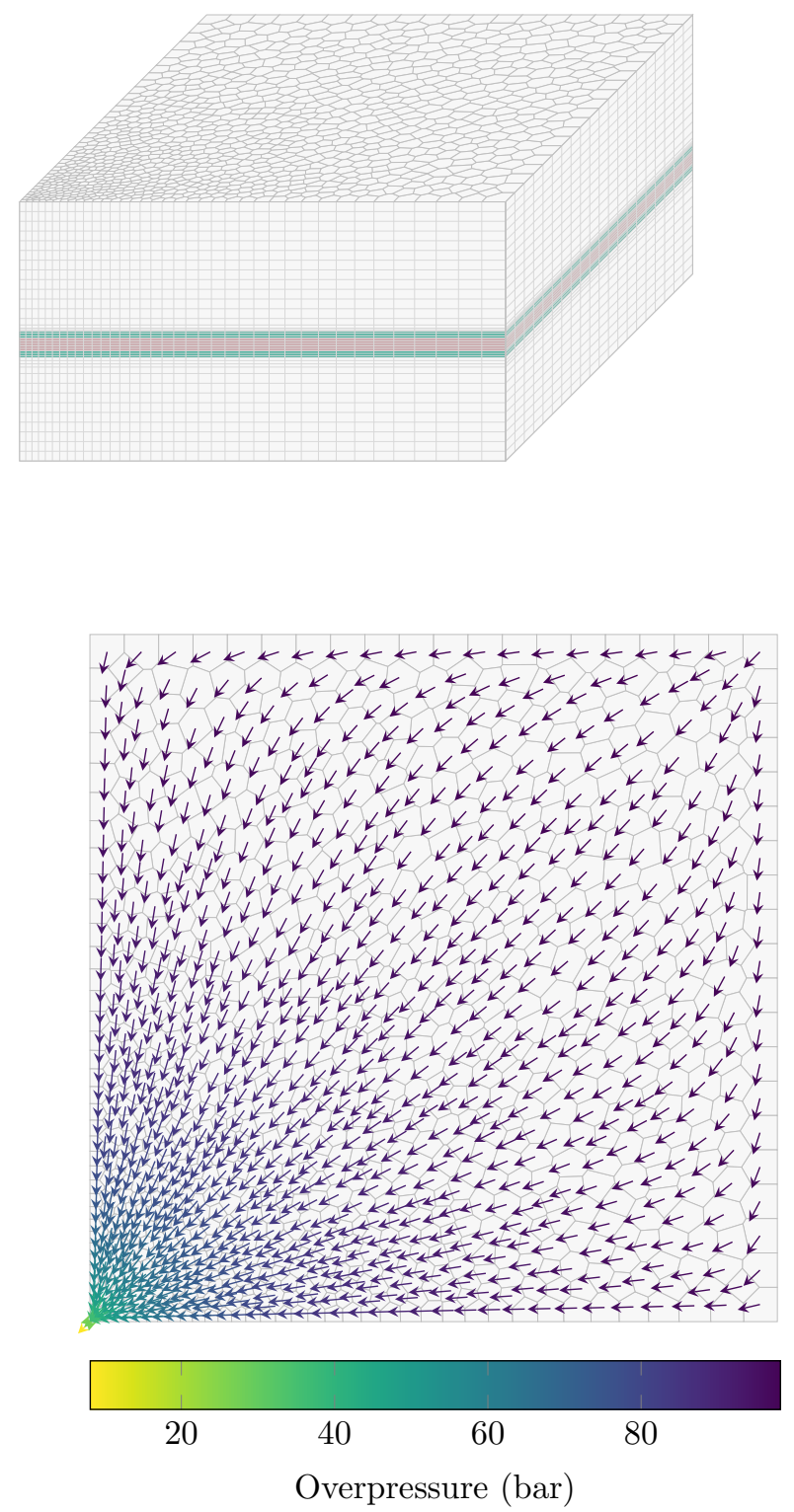

Fig. 6 Horizontal slice of the media inside the reservoir $(z=-2200 m)$. The arrows represent the fluid velocity and are colored from the pressure values computed in the cells.

been significantly modified. As shown on figure 7 where the data are plotted on two vertical lines, the first one being located in the cell in contact with the well and the second one being located in the middle of the $(x, y)$ plane. The horizontal stress $\sigma_{y y}$ is equal to the stress $\sigma_{x x}$ because of symmetry and is therefore not represented. Note that this time, the absolute pressure has been plotted, which allows to read the effective stress $\bar{\sigma}^{e}$ as the difference between the curves.

This effective stress drop around the well leads to the strain and compaction of the reservoir. The initial vertical displacement $\mathbf{u}_{z}^{0}$ due to gravity has been subtracted to the final displacement to highlight the effect of the fluid production. The resulting field is represented on figure 8 . The 


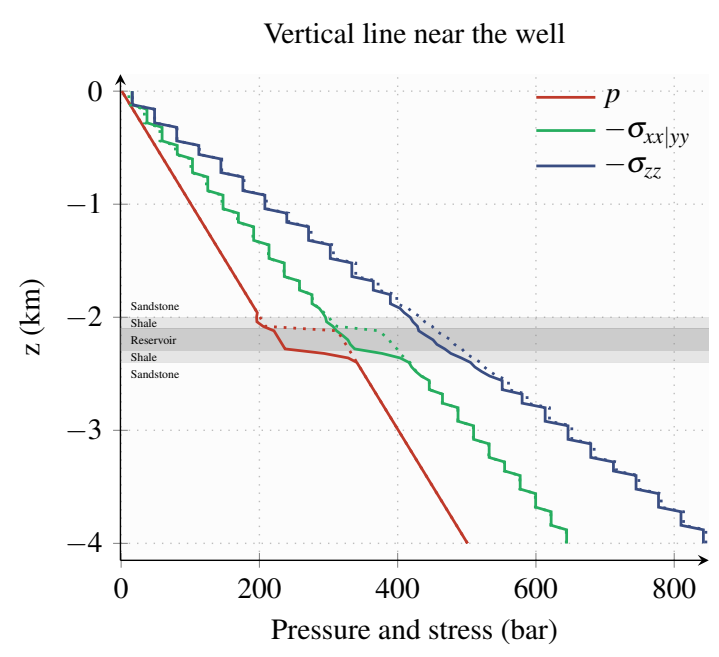

Vertical line in the middle of the domain

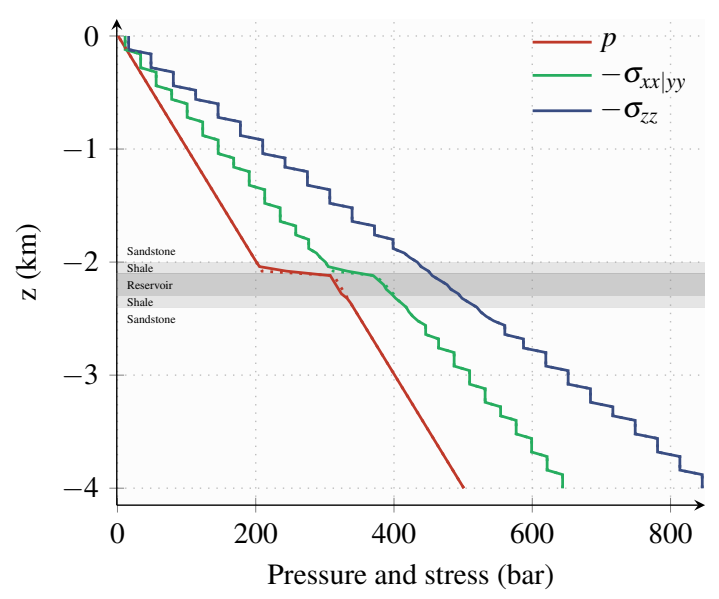

Fig. 7 Evolution of the stress along a vertical line near the well (top) or in the middle of the domain (bottom). The data are plotted at initial (dotted line) and final (solid line) time.

maximum value of vertical displacement $\mathbf{u}_{z}$ is about one meter which is a realistic value. One more thing to note is the flexing of the overburden generated by the subsidence. It can be seen on figure 8 where the horizontal displacement $\mathbf{u}_{x}$ is plotted on the lateral boundary plane $y=0$. Once again, a symmetric behaviour is visually observed for the horizontal displacement $\mathbf{u}_{y}$ on the lateral plane $x=0$, and this even if the mesh is not geometrically symmetric.

\section{Conclusions}

In this work, we made use of the recent improvements about the virtual element methods in order to discretize the mechanical equilibrium equation appearing in the Biot poroelastic model. We employed the simplest flux approximation for the finite volume method to deal with the fluid conservation equation, obtaining a fully coupled scheme for which we detailed the computation of the local matrices and the assembling procedure. We provided a mathematical analysis of this scheme including a bound on the approximation error, and showed numerical results illustrating this error estimate and the ability of the scheme to treat realistic cases. The numerical scheme could be improved in several ways. From the point of view of spatial discretization, other finite volume schemes could and should indeed be used to remove the orthogonality requirement on the mesh. From the point of view of performances, some iterative algorithms may be investigated for the resolution of the coupled system. Finally, from the point of view of the physical modelling, more elaborated mechanical laws such as for example plasticity could be used to provide a better description of the mechanical deformation. Nevertheless, we demonstrated the ability of our scheme to work with Voronoï grids, even in the context of realistic settings. As the extension to more general shapes is not so far, we hope this work will help the generalisation of the use of polyhedral grids: the flexibility in the meshing of subsurface geometries would be improved.

\section{References}

1. Ahmad, B., Alsaedi, A., Brezzi, F., Marini, L., Russo, A.: Equivalent projectors for virtual element methods. Computers and Mathematics with Applications 66(3), 376-391 (2013). DOI 10.1016/j.camwa.2013.05.015

2. Andersen, O., Nilsen, H.M., Raynaud, X.: Virtual element method for geomechanical simulations of reservoir models. Computational Geosciences 21(5), 877-893 (2017). DOI 10.1007/ s10596-017-9636-1

3. Beirão Da Veiga, L., Brezzi, F., Cangiani, A., Manzini, G., Marini, L., Russo, A.: Basic principles of virtual element methods. Mathematical Models \& Methods in applied sciences 23(1), 199-214 (2013). DOI 10.1142/S0218202512500492

4. Beirão Da Veiga, L., Brezzi, F., Marini, L.: Virtual elements for linear elasticity problems. SIAM Journal on Numerical Analysis 51(2), 794-812 (2013). DOI 10.1137/120874746

5. Beirão Da Veiga, L., Brezzi, F., Marini, L., Russo, A.: The hitchhiker's guide to the virtual element method. Mathematical Models \& Methods in applied sciences 24(8), 1541-1573 (2014). DOI 10.1142/S021820251440003X

6. Beirão Da Veiga, L., Lovadina, C., Mora, D.: A virtual element method for elastic and inelastic problems on polytope meshes. Computer Methods in Applied Mechanics and Engineering 295, 327 - 346 (2015). DOI 10.1016/j.cma.2015.07.013

7. Beiro Da Veiga, L., Brezzi, F., Marini, L., Russo, A.: Serendipity nodal VEM spaces. Computers \& Fluids 141, 2-12 (2016). DOI 10.1016/j.compfluid.2016.02.015

8. Bellomo, N., Brezzi, F., Manzini, G.: Recent techniques for pde discretizations on polyhedral meshes. Mathematical Models and Methods in Applied Sciences 24(08), 1453-1455 (2014). DOI 10.1142/S0218202514030018

9. Berrone, S., Borio, A.: Orthogonal polynomials in badly shaped polygonal elements for the virtual element method. Finite Elements in Analysis and Design 129, 14 - 31 (2017). DOI 10.1016/j.finel.2017.01.006

10. Biot, M.A.: General theory of three-dimensional consolidation. Journal of Applied Physics 12(2), 155-164 (1941). DOI 10.1063/ 1.1712886 

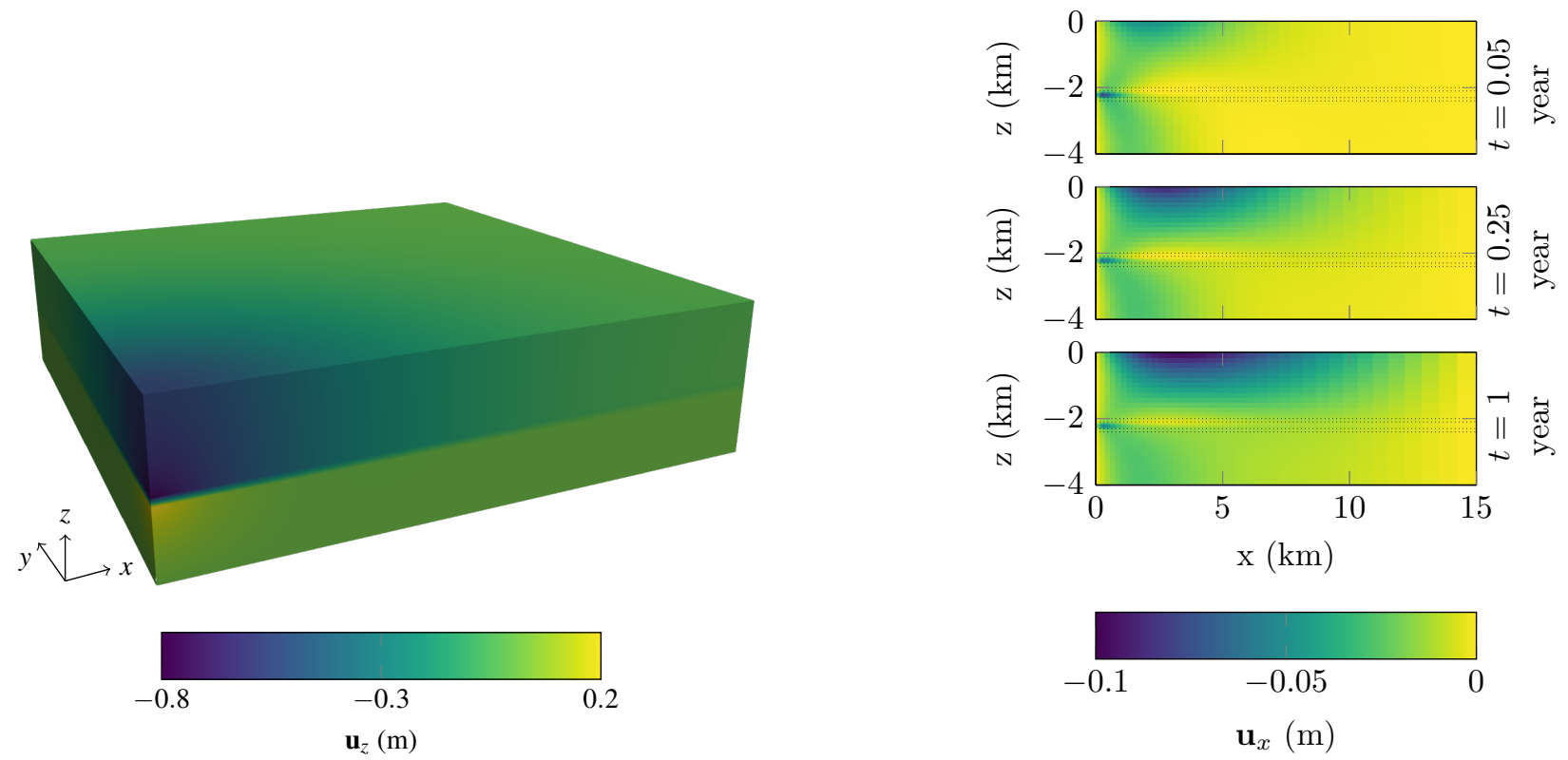

Fig. 8 Vertical displacement at the final time on the whole domain (left) and horizontal displacement at different time steps on lateral slice (right). Dotted lines delimit the physical layers.

11. Bishop, J.: A displacement-based finite element formulation for general polyhedra using harmonic shape functions. International Journal for Numerical Methods in Engineering 97(1), 1-31 (2014). DOI 10.1002/nme.4562

12. Brenner, S.C., Qingguang, G., Sung, L.: Some estimates for virtual element methods. Computational Methods in Applied Mathematics 17(4), 553-574 (2017). DOI 10.1515/cmam-2017-0008

13. Brenner, S.C., Scott, L.R.: The Mathematical Theory of Finite Element Methods, Texts in Applied Mathematics, vol. 15, 3 edn. Springer (2008). DOI 10.1007/978-0-387-75934-0

14. Cangiani, A., Georgoulis, E.H., Pryer, T., Sutton, O.J.: A posteriori error estimates for the virtual element method. Numerische Mathematik 137(4), 857-893 (2017). DOI 10.1007/ s00211-017-0891-9

15. Cockburn, B., Gopalakrishnan, J., Lazarov, R.: Unified hybridization of discontinuous Galerkin, mixed, and continuous Galerkin methods for second order elliptic problems. SIAM Journal on Numerical Analysis 47(2), 1319-1365 (2009). DOI 10.1137/ 070706616

16. Di Pietro, D.A., Ern, A., Lemaire, S.: A Review of Hybrid HighOrder Methods: Formulations, Computational Aspects, Comparison with Other Methods, pp. 205-236. Springer (2016). DOI 10.1007/978-3-319-41640-3_7

17. Droniou, J.: Finite volume schemes for diffusion equations: Introduction to and review of modern methods. Mathematical Models \& Methods in Applied Sciences 24(8, SI), 1575-1619 (2014). DOI 10.1142/S0218202514400041

18. Eymard, R., Gallouët, T., Herbin, R.: Finite volume methods. In: Techniques of Scientific Computing (Part 3), Handbook of $\mathrm{Nu}$ merical Analysis, vol. 7, pp. 713 - 1018. Elsevier (2000). DOI 10.1016/S1570-8659(00)07005-8

19. Gain, A.L., Talischi, C., Paulino, G.H.: On the virtual element method for three-dimensional linear elasticity problems on arbitrary polyhedral meshes. Computer Methods in Applied Mechanics and Engineering 282, 132 - 160 (2014). DOI 10.1016/j.cma. 2014.05.005

20. Guy, N., Enchéry, G., Renard, G.: Numerical modeling of thermal eor: Comprehensive coupling of an amr-based model of thermal fluid flow and geomechanics. Oil Gas Sci. Technol. - Rev. IFP
Energies nouvelles 67(6), 1019-1027 (2012). DOI 10.2516/ogst/ 2012052

21. Lemaire, S.: Nonconforming discretizations of a poromechanical model on general meshes. Ph.D. thesis, Université Paris-Est (2013)

22. Paulino, G.H., Gain, A.L.: Bridging art and engineering using Escher-based virtual elements. Structural and Multidisciplinary Optimization 51(4), 867-883 (2015). DOI 10.1007/ s00158-014-1179-7

23. Quey, R., Dawson, P., Barbe, F.: Large-scale 3d random polycrystals for the finite element method: Generation, meshing and remeshing. Computer Methods in Applied Mechanics and Engineering 200, 1729-1745 (2011). DOI 10.1016/j.cma.2011.01.002

24. Rivière, B., Wheeler, M.F., Girault, V.: A priori error estimates for finite element methods based on discontinuous approximation spaces for elliptic problems. SIAM Journal on Numerical Analysis 39(3), 902-931 (2002)

25. Settari, A., Mourits, F.: Coupling of geomechanics and reservoir simulation models. In: Proceedings, 8th International Conference on Computer Methods and Advances in Geomechanics, vol. 3, pp. 2151-2158. Balkema (1994)

26. Showalter, R.: Diffusion in poro-elastic media. Journal of Mathematical Analysis and Applications 251(1), 310 - 340 (2000). DOI 10.1006/jmaa.2000.7048

27. Sukumar, N., Malsch, E.: Recent advances in the construction of polygonal finite element interpolants. Archives of Computational Methods in Engineering 13(1), 129 (2006). DOI 10.1007/ BF02905933

28. Beiro da Veiga, L., Lipnikov, K., Manzini, G.: The Mimetic Finite Difference Method for Elliptic Problems, Modeling, Simulation \& Applications, vol. 11. Springer (2014). DOI 10.1007/ 978-3-319-02663-3

29. Wang, J., Ye, X.: A weak Galerkin mixed finite element method for second order elliptic problems. Mathematics of Computation 83(289), 2101-2126 (2014). DOI 10.1090/ S0025-5718-2014-02852-4

30. Zoback, M.D.: Reservoir Geomechanics. Cambridge University Press (2007). DOI 10.1017/CBO9780511586477 


\section{A Appendix: details on the proofs}

We provide here the details of the computation that led to the error estimate.

\section{A.1 Proof of the tools}

1. For two real $a$ and $b$ and for some $\varepsilon>0$,

$$
\left(\sqrt{\varepsilon} a-\frac{b}{\sqrt{\varepsilon}}\right)^{2}=\varepsilon a^{2}-2 a b+\frac{b^{2}}{\varepsilon} \geq 0
$$

implying that $2 a b \leq \varepsilon a^{2}+\frac{b^{2}}{\varepsilon}$.

2. Let $\mathbf{v} \in\left(H^{1}(\Omega)\right)^{d}$. Since $\operatorname{div}(\mathbf{v})=\sum_{k=1}^{d} \frac{\partial \mathbf{v}_{k}}{\partial k}$, we have

$$
\begin{aligned}
\|\operatorname{div}(\mathbf{v})\|_{L^{2}(\Omega)} & =\left\|\sum_{k=1}^{d} \frac{\partial \mathbf{v}_{k}}{\partial k}\right\|_{L^{2}(\Omega)} \\
& \leq \sum_{k=1}^{d}\left\|\frac{\partial \mathbf{v}_{k}}{\partial k}\right\|_{L^{2}(\Omega)} \text { (Triangle inequality) } \\
& \leq\left(\sum_{k=1}^{d} 1\right)^{\frac{1}{2}}\left(\sum_{k=1}^{d}\left\|\frac{\partial \mathbf{v}_{k}}{\partial k}\right\|_{L^{2}(\Omega)}^{2}\right)^{\frac{1}{2}} \text { (Cauchy-Schwarz) } \\
& \leq \sqrt{d}|\mathbf{v}|_{H^{1}(\Omega)} .
\end{aligned}
$$

3. Apply Taylor's expansion with integral remainder

$v(t)=\sum_{k=0}^{n} \frac{f^{(k)}(a)}{k !}(t-a)^{k}+\int_{a}^{t} \frac{f^{(n+1)}(s)}{n !}(t-s)^{n} d s$

with $t=t^{n}, a=t^{n-1}$ and $n=0$ for the first equality or $n=1$ for the second one.

4. Let $\mathscr{B}$ be a symmetric bilinear form and $\left\{a_{n}\right\}_{n \geq 0},\left\{b_{n}\right\}_{n \geq 0}$ two sequences belonging to the space of its arguments. For $N=1$ we obviously have $\mathscr{B}\left(a_{1}, b_{1}-b_{0}\right)=\mathscr{B}\left(a_{1}, b_{1}\right)-\mathscr{B}\left(a_{1}, b_{0}\right)$. Now assume that

$$
\begin{aligned}
\sum_{k=1}^{N} \mathscr{B}\left(a_{k}, b_{k}-b_{k-1}\right) & =\mathscr{B}\left(a_{N}, b_{N}\right)-\mathscr{B}\left(a_{1}, b_{0}\right) \\
& -\sum_{k=1}^{N-1} \mathscr{B}\left(b_{k}, a_{k+1}-a_{k}\right) \text { holds for a given } \mathrm{N} .
\end{aligned}
$$

Then

$$
\begin{aligned}
& \sum_{k=1}^{N+1} \mathscr{B}\left(a_{k}, b_{k}-b_{k-1}\right)=\mathscr{B}\left(a_{N+1}, b_{N+1}-b_{N}\right) \\
& +\sum_{k=1}^{N} \mathscr{B}\left(a_{k}, b_{k}-b_{k-1}\right) \\
& =\mathscr{B}\left(a_{N+1}, b_{N+1}-b_{N}\right)+\mathscr{B}\left(a_{N}, b_{N}\right)-\mathscr{B}\left(a_{1}, b_{0}\right) \\
& -\sum_{k=1}^{N-1} \mathscr{B}\left(b_{k}, a_{k+1}-a_{k}\right) \text { by assumption } \\
& =\mathscr{B}\left(a_{N+1}, b_{N+1}\right)-\mathscr{B}\left(a_{N+1}, b_{N}\right)+\mathscr{B}\left(a_{N}, b_{N}\right) \\
& =\mathscr{B}\left(a_{N+1}, b_{N+1}\right)-\mathscr{B}\left(a_{1}, b_{0}\right) \\
& -\mathscr{B}\left(a_{1}, b_{0}\right)-\sum_{k=1}^{N-1} \mathscr{B}\left(b_{k}, a_{k+1}-a_{k}\right) \\
& -\sum_{k=1}^{N} \mathscr{B}\left(b_{k}, a_{k+1}-a_{k}\right) \text { by symmetry of } \mathscr{B} \text {. }
\end{aligned}
$$

5. For two sequences $\left\{a_{n}\right\}_{n \geq 0},\left\{b_{n}\right\}_{n \geq 0}$ in $L^{2}(\Omega)$ or in $\left(L^{2}(\Omega)\right)^{d}$ and $n \geq 1$,

$$
\begin{aligned}
& \left|\int_{\Omega}\left(b^{n}-b^{n-1}\right) a^{n}\right|=\int_{\Omega}\left|\left(\int_{t^{n-1}}^{t^{n}} \partial_{t} b(s) d s\right)\right|\left|a^{n}\right| \\
& \quad \leq \int_{\Omega}\left(\Delta t \int_{t^{n-1}}^{t^{n}}\left(\partial_{t} b(s)\right)^{2} d s\right)^{\frac{1}{2}}\left|a^{n}\right|(\mathrm{CS} \text { in time }) \\
& \quad \leq \sqrt{\Delta t}\left(\int_{\Omega} \int_{t^{n-1}}^{t^{n}}\left(\partial_{t} b(s)\right)^{2} d s\right)^{\frac{1}{2}}\left(\int_{\Omega}\left(a^{n}\right)^{2}\right)^{\frac{1}{2}}(\mathrm{CS} \text { in space }) \\
& \quad=\left\|a^{n}\right\|_{L^{2}} \sqrt{\Delta t}\left(\int_{t^{n-1}}^{t^{n}} \int_{\Omega}\left(\partial_{t} b(s)\right)^{2} d s\right)^{\frac{1}{2}} \\
& \quad=\left\|a^{n}\right\|_{L^{2}}\left(\Delta t \int_{t^{n-1}}^{t^{n}}\left\|\partial_{t} b(s)\right\|_{L^{2}}^{2} d s\right)^{\frac{1}{2}} .
\end{aligned}
$$

\section{A.2 Proof of Grönwall's inequality}

We provide the proof for a more general inequality:

Lemma 6 Let $\left(a_{n}\right)_{n \geq 0},\left(b_{n}\right)_{n \geq 0},\left(c_{n}\right)_{n \geq 0}$ be sequences of nonnegative numbers with $\left(c_{n}\right)_{n \geq 0}$ non decreasing. Assume that

$a_{0}+b_{0} \leq c_{0}$

and that there exists $\lambda>0$ such that

$\forall N \geq 1, \quad a_{N}+b_{N} \leq \lambda \sum_{m=0}^{N-1} a_{m}+c_{N}$

Then there holds

$\forall N \geq 0, \quad a_{N}+b_{N} \leq c_{N} \exp (\lambda N)$.

We first proceed by induction, showing that

$\forall N \geq 0, \quad a_{N}+b_{N} \leq c_{N}(1+\lambda)^{N}$

By assumption, for $N=0$ we have $a_{0}+b_{0} \leq c_{0}$. Now assume that the property holds for every $n \leq N$ and lets prove it holds at range $N+1$. Using the assumptions of the lemma,

$$
\begin{aligned}
a_{N+1}+b_{N+1} & \leq \lambda \sum_{m=0}^{N} a_{m}+c_{N+1} \\
& \leq \lambda \sum_{m=0}^{N}\left(c_{m}(1+\lambda)^{m}-b_{m}\right)+c_{N+1} \quad \text { from }(\star) \\
& \leq \lambda c_{N+1} \sum_{m=0}^{N}(1+\lambda)^{m}+c_{N+1}
\end{aligned}
$$

because $\left(c_{n}\right)_{n \geq 0}$ is non decreasing and $\left(b_{n}\right)_{n \geq 0}$ is nonnegative. Summing a geometric sequence,

$\sum_{m=0}^{N}(1+\lambda)^{m}=\frac{1-(1+\lambda)^{N+1}}{1-(1+\lambda)}=\frac{(1+\lambda)^{N+1}-1}{\lambda}$

and thus $a_{N+1}+b_{N+1} \leq c_{N+1}(1+\lambda)^{N+1}$. This proves the induction, and the lemma comes from the estimate $(1+\lambda)^{n} \leq \exp (n \lambda)$ 


\section{A.3 Proof of the estimates}

We provide here the proofs of the estimates obtained on the $B_{i}$ terms.

- $\Delta t B_{1}^{n}=-\alpha \Delta t \int_{\Omega} \operatorname{div}\left(D^{-} E_{\mathbf{u}}^{I, n}\right) E_{p}^{A, n}+\alpha \Delta t \int_{\Omega} \operatorname{div}\left(D^{-} E_{\mathbf{u}}^{A, n}\right) E_{p}^{I, n}$

We split this term in two parts corresponding to the two integrals. For the first part, applying successively tool 5 and tool 2,

$$
\begin{aligned}
\left|\Delta t \int_{\Omega} E_{p}^{A, n} \operatorname{div}\left(D^{-} E_{\mathbf{u}}^{I, n}\right)\right| & =\sqrt{\Delta t}\left\|E_{p}^{A, n}\right\|_{L^{2}}\left(\int_{t^{n-1}}^{t^{n}}\left\|\operatorname{div}\left(\partial_{t} E_{\mathbf{u}}^{I}\right)\right\|_{L^{2}}^{2}\right)^{\frac{1}{2}} \\
& \leq \sqrt{d \Delta t}\left\|E_{p}^{A, n}\right\|_{L^{2}}\left(\int_{t^{n-1}}^{t^{n}}\left\|\partial_{t} E_{\mathbf{u}}^{I}\right\|_{H^{1}}^{2}\right)^{\frac{1}{2}}
\end{aligned}
$$

then we apply Young's inequality with $\varepsilon=\frac{1}{4}$ for the last term and $\varepsilon=\frac{\varepsilon_{r}}{4}$ for the others:

$$
\begin{gathered}
\left|2 \sum_{n=1}^{N} \alpha \Delta t \int_{\Omega} E_{p}^{A, n} \operatorname{div}\left(D^{-} E_{\mathbf{u}}^{I, n}\right)\right| \leq \frac{\varepsilon_{r}}{4} \sum_{n=1}^{N-1} \Delta t\left\|E_{p}^{A, n}\right\|_{L^{2}}^{2}+\frac{\Delta t}{4}\left\|E_{p}^{A, N}\right\|_{L^{2}}^{2} \\
+4 d \alpha^{2}\left(\frac{1}{\varepsilon_{r}} \sum_{n=1}^{N-1}\left(\int_{t^{n-1}}^{t^{n}}\left\|\partial_{t} E_{\mathbf{u}}^{I}\right\|_{H^{1}}^{2}\right)^{\frac{1}{2}}+\left(\int_{t^{N-1}}^{t^{N}}\left\|\partial_{t} E_{\mathbf{u}}^{I}\right\|_{H^{1}}^{2}\right)^{\frac{1}{2}}\right) .
\end{gathered}
$$

We now define the $L^{2}$ in time norm as $\|g\|_{L^{2}(T)}^{2}=\int_{t^{0}}^{t^{N}} g(s)^{2} d s$. We can add the missing terms (which are non negative) to write

$\left|2 \sum_{n=1}^{N} \alpha \Delta t \int_{\Omega} E_{p}^{A, n} \operatorname{div}\left(D^{-} E_{\mathbf{u}}^{I, n}\right)\right| \leq \frac{\varepsilon_{r}}{4} \sum_{n=1}^{N-1} \Delta t\left\|E_{p}^{A, n}\right\|_{L^{2}}^{2}+\frac{\Delta t}{4}\left\|E_{p}^{A, N}\right\|_{L^{2}}^{2}$

$+4 d \alpha^{2}\left(\frac{1}{\varepsilon_{r}}\left\|\partial_{t} E_{\mathbf{u}}^{I}\right\|_{L^{2}\left(0, T ; H^{1}\right)}^{2}+\left\|\partial_{t} E_{\mathbf{u}}^{I}\right\|_{L^{2}\left(0, T ; H^{1}\right)}^{2}\right)$

$\leq \frac{\varepsilon_{r}}{4} \sum_{n=1}^{N-1} \Delta t\left\|E_{p}^{A, n}\right\|_{L^{2}}^{2}+\frac{\Delta t}{4}\left\|E_{p}^{A, N}\right\|_{L^{2}}^{2}+4 d \alpha^{2}\left(\frac{1}{\tilde{\varepsilon}_{r}}\left\|\partial_{t} E_{\mathbf{u}}^{I}\right\|_{L^{2}\left(0, T ; H^{1}\right)}^{2}\right)$

where $\tilde{\varepsilon}_{r}=\frac{\varepsilon_{r}}{1+\varepsilon_{r}}$. For the second part, a partial summation is done first

$$
\begin{aligned}
2 \sum_{n=1}^{N} \Delta t & \int_{\Omega} \alpha \operatorname{div}\left(D^{-} E_{\mathbf{u}}^{A, n}\right) E_{p}^{I, n}=2 \int_{\Omega} \alpha \operatorname{div}\left(E_{\mathbf{u}}^{A, N}\right) E_{p}^{I, N} \\
& -2 \int_{\Omega} \alpha \operatorname{div}\left(E_{\mathbf{u}}^{A, 0}\right) E_{p}^{I, 1}-2 \sum_{n=1}^{N-1} \Delta t \int_{\Omega} \alpha \operatorname{div}\left(E_{\mathbf{u}}^{A, n}\right) D^{-} E_{p}^{I, n+1}
\end{aligned}
$$

and, using the same procedure as above,

$$
\begin{aligned}
2\left|\int_{\Omega} \alpha \operatorname{div}\left(E_{\mathbf{u}}^{A, N}\right) E_{p}^{I, N}\right| & \leq \frac{\varepsilon_{l}}{4}\left\|E_{\mathbf{u}}^{A, N}\right\|_{H^{1}}^{2}+\frac{4 d \alpha^{2}}{\varepsilon_{l}}\left\|E_{p}^{I, N}\right\|_{L^{2}}^{2}, \\
2\left|\int_{\Omega} \alpha \operatorname{div}\left(E_{\mathbf{u}}^{A, 0}\right) E_{p}^{I, 1}\right| & \leq \frac{\varepsilon_{l}}{4}\left\|E_{\mathbf{u}}^{A, 0}\right\|_{H^{1}}^{2}+\frac{4 d \alpha^{2}}{\varepsilon_{l}}\left\|E_{p}^{I, 1}\right\|_{L^{2}}^{2},
\end{aligned}
$$

$2\left|\sum_{n=1}^{N-1} \alpha \Delta t \int_{\Omega} \operatorname{div}\left(E_{\mathbf{u}}^{A, n}\right) D^{-} E_{p}^{I, n+1}\right| \leq \frac{\varepsilon_{r}}{4} \sum_{n=1}^{N-1} \Delta t\left\|E_{\mathbf{u}}^{A, n}\right\|_{H^{1}}^{2}$

$$
+\frac{4 d \alpha^{2}}{\varepsilon_{r}}\left\|\partial_{t} E_{p}^{I}\right\|_{L^{2}\left(0, T ; L^{2}\right)}^{2} .
$$

Combining the two parts leads to the estimation on $B_{1}$ :

$$
\begin{aligned}
& \left|2 \Delta t \sum_{n=1}^{N} B_{1}\right| \leq \frac{\varepsilon_{r}}{4} \sum_{n=1}^{N-1} \Delta t\left\|E_{p}^{A, n}\right\|_{L^{2}}^{2}+\frac{\varepsilon_{r}}{4} \sum_{n=1}^{N-1} \Delta t\left\|E_{\mathbf{u}}^{A, n}\right\|_{H^{1}}^{2} \\
& +\frac{\Delta t}{4}\left\|E_{p}^{A, N}\right\|_{L^{2}}^{2}+\frac{\varepsilon_{l}}{4}\left\|E_{\mathbf{u}}^{A, N}\right\|_{H^{1}}^{2}+\frac{\varepsilon_{l}}{4}\left\|E_{\mathbf{u}}^{A, 0}\right\|_{H^{1}}^{2} \\
& +4 d \alpha^{2}\left(\frac{\left\|\partial_{t} E_{\mathbf{u}}^{I}\right\|_{L^{2}\left(0, T ; H^{1}\right)}^{2}}{\tilde{\varepsilon}_{r}}+\frac{\left\|E_{p}^{I, N}\right\|_{L^{2}}^{2}}{\varepsilon_{l}}+\frac{\left\|E_{p}^{I, 1}\right\|_{L^{2}}^{2}}{\varepsilon_{l}}+\frac{\left\|\partial_{t} E_{p}^{I}\right\|_{L^{2}\left(0, T ; L^{2}\right)}^{2}}{\varepsilon_{r}}\right)
\end{aligned}
$$

$\bullet \Delta t B_{2}^{n}=\Delta t \sum_{K \in \tau_{h}} a^{K}\left(-E_{\mathbf{u}}^{\pi, n}, D^{-} E_{\mathbf{u}}^{A, n}\right)+\Delta t \sum_{K \in \tau_{h}} a_{h}\left(E_{\mathbf{u}}^{\pi, n}-E_{\mathbf{u}}^{I, n}, D^{-} E_{\mathbf{u}}^{A, n}\right)$

We start with the $a^{K}$ part. We denote $M_{K}$ the continuity constant of $a^{K}$ and we note that, for a function $\mathbf{u}$ locally in $\left(H^{1}(K)\right)^{d}$ and a function $\mathbf{v}$ globally in $\left(H^{1}(\Omega)\right)^{d}$ and for some $\varepsilon>0$,

$$
\begin{aligned}
\left|\sum_{K \in \tau_{h}} a^{K}(\mathbf{u}, \mathbf{v})\right| & \leq \sum_{K \in \tau_{h}}\left(M_{K}\|\mathbf{u}\|_{H^{1}(K)}\|\mathbf{v}\|_{H^{1}(K)}\right) \\
& \leq \sum_{K \in \tau_{h}}\left(\frac{M_{K}^{2}}{2 \varepsilon}\|\mathbf{u}\|_{H^{1}(K)}^{2}+\frac{\varepsilon}{2}\|\mathbf{v}\|_{H^{1}(K)}^{2}\right) \\
& \leq \frac{M^{2}}{2 \varepsilon} \sum_{K \in \tau_{h}}\|\mathbf{u}\|_{H^{1}(K)}^{2}+\frac{\varepsilon}{2} \sum_{K \in \tau_{h}}\|\mathbf{v}\|_{H^{1}(K)}^{2} \\
& =\frac{M^{2}}{2 \varepsilon}\|\mathbf{u}\|_{H^{1}(\tau)}^{2}+\frac{\varepsilon}{2}\|\mathbf{v}\|_{H^{1}(\Omega)}^{2}
\end{aligned}
$$

where $M=\max _{K} M_{K}$. We now use the partial summation (recall that $a^{K}$ is symmetric)

$$
\begin{aligned}
& 2 \sum_{n=1}^{N} \Delta t \sum_{K \in \tau_{h}} a^{K}\left(-E_{\mathbf{u}}^{\pi, n}, D^{-} E_{\mathbf{u}}^{A, n}\right)=2 \sum_{K \in \tau_{h}} a^{K}\left(-E_{\mathbf{u}}^{\pi, N}, E_{\mathbf{u}}^{A, N}\right) \\
& -2 \sum_{K \in \tau_{h}} a^{K}\left(-E_{\mathbf{u}}^{\pi, 1}, E_{\mathbf{u}}^{A, 0}\right)-2 \sum_{n=1}^{N-1} \sum_{K \in \tau_{h}} a^{K}\left(-\left(E_{\mathbf{u}}^{\pi, n+1}-E_{\mathbf{u}}^{\pi, n}\right), E_{\mathbf{u}}^{A, n}\right)
\end{aligned}
$$

and combine it with the previous estimate to write

$$
\begin{aligned}
\left|2 \sum_{K \in \tau_{h}} a^{K}\left(-E_{\mathbf{u}}^{\pi, N}, E_{\mathbf{u}}^{A, N}\right)\right| & \leq \frac{8 M^{2}}{\varepsilon_{l}}\left\|E_{\mathbf{u}}^{\pi, N}\right\|_{H^{1}(\tau)}^{2}+\frac{\varepsilon_{l}}{8}\left\|E_{\mathbf{u}}^{A, N}\right\|_{H^{1}}^{2}, \\
\left|2 \sum_{K \in \tau_{h}} a^{K}\left(-E_{\mathbf{u}}^{\pi, 1}, E_{\mathbf{u}}^{A, 0}\right)\right| & \leq \frac{8 M^{2}}{\varepsilon_{l}}\left\|E_{\mathbf{u}}^{\pi, 1}\right\|_{H^{1}(\tau)}^{2}+\frac{\varepsilon_{l}}{8}\left\|E_{\mathbf{u}}^{A, 0}\right\|_{H^{1}}^{2} .
\end{aligned}
$$

For the third term, we also follow the idea of tool 5:

$$
\begin{aligned}
& 2 \sum_{K \in \tau_{h}}\left|a^{K}\left(E_{\mathbf{u}}^{\pi, n+1}-E_{\mathbf{u}}^{\pi, n}, E_{\mathbf{u}}^{A, n}\right)\right|=2 \sum_{K \in \tau_{h}}\left|a^{K}\left(\int_{t^{n}}^{t^{n+1}} \partial_{t} E_{\mathbf{u}}^{\pi}, E_{\mathbf{u}}^{A, n}\right)\right| \\
& \leq 2 \sum_{K \in \tau_{h}} M_{K}\left\|\int_{t^{n}}^{t^{n+1}} \partial_{t} E_{\mathbf{u}}^{\pi}\right\|_{H^{1}(K)}\left\|E_{\mathbf{u}}^{A, n}\right\|_{H^{1}(K)} \\
& \leq 2 \sum_{K \in \tau_{h}} M_{K}\left(\Delta t \int_{t^{n}}^{t^{n+1}}\left\|\partial_{t} E_{\mathbf{u}}^{\pi}\right\|_{H^{1}(K)}^{2}\right)^{\frac{1}{2}}\left\|E_{\mathbf{u}}^{A, n}\right\|_{H^{1}(K)} \\
& \leq \sum_{K \in \tau_{h}} \frac{8 M^{2}}{\varepsilon_{r}} \int_{t^{n}}^{t^{n+1}}\left\|\partial_{t} E_{\mathbf{u}}^{\pi}\right\|_{H^{1}(K)}^{2}+\Delta t \frac{\varepsilon_{r}}{8}\left\|E_{\mathbf{u}}^{A, n}\right\|_{H^{1}(K)}^{2} \\
& =\frac{8 M^{2}}{\varepsilon_{r}} \int_{t^{n}}^{t^{n+1}}\left\|\partial_{t} E_{\mathbf{u}}^{\pi}\right\|_{H^{1}(\tau)}^{2}+\Delta t \frac{\varepsilon_{r}}{8}\left\|E_{\mathbf{u}}^{A, n}\right\|_{H^{1}}^{2} .
\end{aligned}
$$

We now apply the same methods to the $a_{h}$ part, denoting $\tilde{M}_{K}$ the continuity constant of $a_{h}^{K}$ and $\tilde{M}=\max _{K} M_{K}$,

$$
\begin{aligned}
& 2 \sum_{n=1}^{N} \Delta t \sum_{K \in \tau_{h}} a_{h}^{K}\left(E_{\mathbf{u}}^{\pi, n}-E_{\mathbf{u}}^{I, n}, D^{-} E_{\mathbf{u}}^{A, n}\right) \\
& =2 \sum_{K \in \tau_{h}} a_{h}^{k}\left(E_{\mathbf{u}}^{\pi, N}-E_{\mathbf{u}}^{I, N}, E_{\mathbf{u}}^{A, N}\right)-2 \sum_{K \in \tau_{h}} a_{h}^{K}\left(E_{\mathbf{u}}^{\pi, 1}-E_{\mathbf{u}}^{I, 1}, E_{\mathbf{u}}^{A, 0}\right) \\
& \quad-2 \sum_{n=1}^{N-1} \sum_{K \in \tau_{h}} a_{h}^{K}\left(\left(E_{\mathbf{u}}^{\pi, n+1}-E_{\mathbf{u}}^{\pi, n}\right)-\left(E_{\mathbf{u}}^{I, n+1}-E_{\mathbf{u}}^{I, n}\right), E_{\mathbf{u}}^{A, n}\right)
\end{aligned}
$$


and we split the errors $E^{I}$ and $E^{\pi}$

$$
\begin{aligned}
2 \sum_{K \in \tau_{h}}\left|a_{h}^{K}\left(E_{\mathbf{u}}^{\pi, N}-E_{\mathbf{u}}^{I, N}, E_{\mathbf{u}}^{A, N}\right)\right| & \leq \frac{8 \tilde{M}^{2}}{\varepsilon_{l}}\left\|E_{\mathbf{u}}^{\pi, N}\right\|_{H^{1}(\tau)}^{2}+\frac{4 \tilde{M}^{2}}{\varepsilon_{l}}\left\|E_{\mathbf{u}}^{I, N}\right\|_{H^{1}}^{2} \\
& +\left(\frac{\varepsilon_{l}}{8}+\frac{\varepsilon_{l}}{4}\right)\left\|E_{\mathbf{u}}^{A, N}\right\|_{H^{1}}^{2}, \\
2 \sum_{K \in \tau_{h}}\left|a_{h}^{K}\left(E_{\mathbf{u}}^{\pi, 1}-E_{\mathbf{u}}^{I, 1}, E_{\mathbf{u}}^{A, 0}\right)\right| & \leq \frac{8 \tilde{M}^{2}}{\varepsilon_{l}}\left\|E_{\mathbf{u}}^{\pi, 1}\right\|_{H^{1}(\tau)}^{2}+\frac{4 \tilde{M}^{2}}{\varepsilon_{l}}\left\|E_{\mathbf{u}}^{I, 1}\right\|_{H^{1}}^{2} \\
& +\left(\frac{\varepsilon_{l}}{8}+\frac{\varepsilon_{l}}{4}\right)\left\|E_{\mathbf{u}}^{A, 0}\right\|_{H^{1}}^{2}, \\
2 \sum_{K \in \tau_{h}} a_{h}^{K}\left|\left(E_{\mathbf{u}}^{\pi, n+1}-E_{\mathbf{u}}^{\pi, n}, E_{\mathbf{u}}^{A, n}\right)\right| & \leq \frac{8 \tilde{M}^{2}}{\varepsilon_{r}} \int_{t^{n}}^{t^{n+1}}\left\|\partial_{t} E_{\mathbf{u}}^{\pi}\right\|_{H^{1}(\tau)}^{2} \\
& +\frac{\varepsilon_{r}}{8} \Delta t\left\|E_{\mathbf{u}}^{A, n}\right\|_{H^{1}}^{2}, \\
2 \sum_{K \in \tau_{h}} a_{h}^{K}\left|\left(E_{\mathbf{u}}^{I, n+1}-E_{\mathbf{u}}^{I, n}, E_{\mathbf{u}}^{A, n}\right)\right| & \leq \frac{4 \tilde{M}^{2}}{\varepsilon_{r}} \int_{t^{n}}^{t^{n+1}}\left\|\partial_{t} E_{\mathbf{u}}^{I}\right\|_{H^{1}}^{2} \\
& +\frac{\varepsilon_{r}}{4} \Delta t\left\|E_{\mathbf{u}}^{A, n}\right\|_{H^{1}}^{2} .
\end{aligned}
$$

We finally obtain the bound

$$
\begin{aligned}
& \left|2 \Delta t \sum_{n=1}^{N} B_{2}\right| \leq \frac{\varepsilon_{r}}{2} \sum_{n=1}^{N-1} \Delta t\left\|E_{\mathbf{u}}^{A, n}\right\|_{H^{1}}^{2}+\frac{\varepsilon_{l}}{2}\left\|E_{\mathbf{u}}^{A, N}\right\|_{H^{1}}^{2}+\frac{\varepsilon_{l}}{2}\left\|E_{\mathbf{u}}^{A, 0}\right\|_{H^{1}}^{2} \\
& +4 \tilde{M}^{2}\left(\frac{\left\|E_{\mathbf{u}}^{I, 1}\right\|_{H^{1}}^{2}}{\varepsilon_{l}}+\frac{\left\|E_{\mathbf{u}}^{I, N}\right\|_{H^{1}}^{2}}{\varepsilon_{l}}+\frac{\left\|\partial_{t} E_{\mathbf{u}}^{I}\right\|_{L^{2}\left(0, T ; H^{1}\right)}^{2}}{\varepsilon_{r}}\right) \\
& +8\left(M^{2}+\tilde{M}^{2}\right)\left(\frac{\left\|E_{\mathbf{u}}^{\pi, 1}\right\|_{H^{1}(\tau)}^{2}}{\varepsilon_{l}}+\frac{\left\|E_{\mathbf{u}}^{\pi, N}\right\|_{H^{1}(\tau)}^{2}}{\varepsilon_{l}}+\frac{\left\|\partial_{t} E_{\mathbf{u}}^{\pi}\right\|_{L^{2}\left(0, T ; H^{1}(\tau)\right)}^{2}}{\varepsilon_{r}}\right)
\end{aligned}
$$

$\bullet \Delta t B_{3}^{n}=\Delta t \int_{\Omega} c_{0}\left(D^{-} p^{n}-\partial_{t} p^{n}\right) E_{p}^{A, n}+\Delta t \int_{\Omega} \alpha \operatorname{div}\left(D^{-} \mathbf{u}^{n}-\partial_{t} \mathbf{u}^{n}\right) E_{p}^{A, n}$

We first use the Taylor's expansion (tool 3)

$$
\begin{array}{rl}
\Delta t B_{3}^{n}=\int_{\Omega} c_{0} \int_{t^{n-1}}^{t^{n}} \partial_{t t} & p(s)\left(t^{n-1}-s\right)^{2} d s E_{p}^{A, n} \\
& +\int_{\Omega} \alpha \operatorname{div}\left(\int_{t^{n-1}}^{t^{n}} \partial_{t t} \mathbf{u}(s)\left(t^{n-1}-s\right)^{2} d s\right) E_{p}^{A, n}
\end{array}
$$

We then follow the idea of the double Cauchy-Schwarz (tool 5), this time using the fact that $\int_{t^{n-1}}^{t^{n}}\left(t^{n-1}-s\right)^{2} d s=\frac{\Delta t^{3}}{3}$. In addition, tool 2 is used to treat the divergence operator. Thus,

$$
\begin{aligned}
\left|\Delta t B_{3}^{n}\right| \leq c_{0}\left\|E_{p}^{A, n}\right\|_{L^{2}} & \left(\frac{\Delta t^{3}}{3} \int_{t_{n-1}}^{t^{n}}\left\|\partial_{t t} p(s)\right\|_{L^{2}}^{2} d s\right)^{\frac{1}{2}} \\
& +\alpha\left\|E_{p}^{A, n}\right\|_{L^{2}}\left(\frac{d \Delta t^{3}}{3} \int_{t_{n-1}}^{t^{n}}\left\|\partial_{t t} \mathbf{u}(s)\right\|_{H^{1}}^{2} d s\right)^{\frac{1}{2}}
\end{aligned}
$$

Following what has been done for the estimate of $B^{1}$, Young's inequality is used with $\varepsilon=\frac{1}{4}$ for the last term and $\varepsilon=\frac{\varepsilon_{r}}{4}$ for the others:

$$
\begin{aligned}
\left|2 \Delta t \sum_{n=1}^{N} B_{3}^{n}\right| & \leq \frac{2 \varepsilon_{r}}{4} \sum_{n=1}^{N-1} \Delta t\left\|E_{p}^{A, n}\right\|_{L^{2}}^{2}+\frac{2 \Delta t}{4}\left\|E_{p}^{A, N}\right\|_{L^{2}}^{2} \\
& +\frac{4}{3} \Delta t^{2}\left(c_{0}^{2} \frac{\left\|\partial_{t t} p\right\|_{L^{2}\left(0, T ; L^{2}\right)}^{2}}{\tilde{\varepsilon}_{r}}+d \alpha^{2} \frac{\left\|\partial_{t t} \mathbf{u}\right\|_{L^{2}\left(0, T ; H^{1}\right)}^{2}}{\tilde{\varepsilon}_{r}}\right)
\end{aligned}
$$

$\bullet \Delta t B_{4}^{n}=\Delta t \int_{\Omega}\left(\mathbf{f}^{n}-\mathbf{f}_{h}^{n}\right) \cdot D^{-} E_{\mathbf{u}}^{A, n}$

We assimilate the difference $\left(\mathbf{f}^{n}-\mathbf{f}_{h}^{n}\right)$ to an interpolation error denoted $E_{\mathbf{f}}^{I, n}$. Using a partial summation,

$$
\begin{aligned}
2 \Delta t \sum_{n=1}^{N} \int_{\Omega} E_{\mathbf{f}}^{I, n} \cdot D^{-} E_{\mathbf{u}}^{A, n}=2 \int_{\Omega} E_{\mathbf{f}}^{I, N} & E_{\mathbf{u}}^{A, N}-\int_{\Omega} 2 E_{\mathbf{f}}^{I, 1} E_{\mathbf{u}}^{A, 0} \\
& -2 \sum_{n=1}^{N-1} \int_{\Omega}\left(E_{\mathbf{f}}^{I, n+1}-E_{\mathbf{f}}^{I, n}\right) \cdot E_{\mathbf{u}}^{A, n} .
\end{aligned}
$$

Using Cauchy-Schwarz and Young inequalities, and treating the third term with tool 5 , we have

$$
\begin{aligned}
2\left|\int_{\Omega} E_{\mathbf{f}}^{I, N} E_{\mathbf{u}}^{A, N}\right| & \leq 2\left\|E_{\mathbf{f}}^{I, N}\right\|_{L^{2}}\left\|E_{\mathbf{u}}^{A, N}\right\|_{L^{2}} \\
& \leq \frac{4}{\varepsilon_{l}}\left\|E_{\mathbf{f}}^{I, N}\right\|_{L^{2}}^{2}+\frac{\varepsilon_{l}}{4}\left\|E_{\mathbf{u}}^{A, N}\right\|_{H^{1}}^{2}, \\
2\left|\int_{\Omega} E_{\mathbf{f}}^{I, 1} E_{\mathbf{u}}^{A, 0}\right| & \leq 2\left\|E_{\mathbf{f}}^{I, 1}\right\|_{L^{2}}\left\|E_{\mathbf{u}}^{A, 0}\right\|_{L^{2}} \\
& \leq \frac{4}{\varepsilon_{l}}\left\|E_{\mathbf{f}}^{I, 1}\right\|_{L^{2}}^{2}+\frac{\varepsilon_{l}}{4}\left\|E_{\mathbf{u}}^{A, 0}\right\|_{H^{1}}^{2}, \\
2\left|\int_{\Omega}\left(E_{\mathbf{f}}^{I, n+1}-E_{\mathbf{f}}^{I, n}\right) \cdot E_{\mathbf{u}}^{A, n}\right| & \leq\left\|E_{\mathbf{u}}^{A, n}\right\|_{L^{2}}\left(\Delta t \int_{t^{n}}^{t^{n+1}}\left\|\partial_{t} E_{\mathbf{f}}^{I}\right\|_{L^{2}}^{2}\right)^{\frac{1}{2}} \\
& \leq \frac{4}{\varepsilon_{r}} \int_{t^{n}}^{t^{n+1}}\left\|\partial_{t} E_{\mathbf{f}}^{I}\right\|_{L^{2}}^{2}+\frac{\varepsilon_{r}}{4} \Delta t\left\|E_{\mathbf{u}}^{A, n}\right\|_{H^{1}}^{2},
\end{aligned}
$$

and thus, noticing that $\left\|E_{\mathbf{u}}^{A}\right\|_{L^{2}} \leq\left\|E_{\mathbf{u}}^{A}\right\|_{H^{1}}$,

$\left|2 \Delta t \sum_{n=1}^{N} B_{4}^{n}\right| \leq \frac{\varepsilon_{r}}{4} \sum_{n=1}^{N-1} \Delta t\left\|E_{\mathbf{u}}^{A, n}\right\|_{H^{1}}^{2}+\frac{\varepsilon_{l}}{4}\left\|E_{\mathbf{u}}^{A, N}\right\|_{H^{1}}^{2}+\frac{4}{\varepsilon_{r}}\left\|\partial_{t} E_{\mathbf{f}}^{I}\right\|_{L^{2}\left(0, T ; L^{2}\right)}^{2}$

$$
+\frac{4}{\varepsilon_{l}}\left(\left\|E_{\mathbf{f}}^{I, N}\right\|_{L^{2}}^{2}+\left\|E_{\mathbf{f}}^{I, 1}\right\|_{L^{2}}^{2}\right)+\frac{\varepsilon_{l}}{4}\left\|E_{\mathbf{u}}^{A, 0}\right\|_{H^{1}}^{2} \square
$$

$\bullet \Delta t B_{5}^{n}=-\Delta t \sum_{K} \sum_{f \subset \partial K}\left(\overline{F_{K f}^{n}}-F_{K f}^{\star, n}\right) E_{p}^{A, n}$

For this part, we use the consistency of the fluxes $\overline{F_{K f}^{n}}=-\overline{F_{L f}^{n}}$ and $F_{K f}^{\star, n}=-F_{L f}^{\star, n}$. This allows to reorganise the double sum in the following way, $K$ standing for any of the two neighboring cells of $f$,

$$
\begin{aligned}
& \sum_{K} \sum_{f \subset \partial K}\left(\overline{F_{K f}^{n}}-F_{K f}^{\star, n}\right) E_{p}^{A, n}=\sum_{f \subset \bar{\Omega} \backslash \Gamma_{N_{p}}}\left(\overline{F_{K f}^{n}}-F_{K f}^{\star, n}\right) D_{f}\left(E_{p}^{A, n}\right) \\
& \leq\left(\sum_{f \subset \bar{\Omega} \backslash \Gamma_{N_{p}}} T_{f}^{-1}\left|\overline{F_{K f}^{n}}-F_{K f}^{\star, n}\right|^{2}\right)^{\frac{1}{2}}\left(\sum_{f \subset \bar{\Omega} \backslash I_{N_{p}}} T_{f} D_{f}\left(E_{p}^{A, n}\right)^{2}\right)^{\frac{1}{2}} \\
& \leq \frac{\left|E_{p}^{A, n}\right|_{1, \tau}^{2}}{2}+\sum_{f \subset \bar{\Omega} \backslash \Gamma_{N_{p}}} \frac{\left|\overline{F_{K f}^{n}}-F_{K f}^{\star, n}\right|^{2}}{2 T_{f}} \text { (Young) }
\end{aligned}
$$

and thus

$$
\begin{aligned}
& \left|2 \Delta t \sum_{n=1}^{N} B_{5}^{n}\right| \leq \sum_{n=1}^{N} \Delta t\left|E_{p}^{A, n}\right|_{1, \tau}^{2}+\sum_{n=1}^{N} \Delta t \sum_{f \subset \bar{\Omega} \backslash \Gamma_{N_{p}}} \frac{\left|\overline{F_{K f}^{n}}-F_{K f}^{\star, n}\right|^{2}}{T_{f}} \\
& \bullet \Delta t B_{6}^{n}=-\Delta t c_{0} \int_{\Omega} E_{p}^{A, n} D^{-} E_{p}^{I, n}
\end{aligned}
$$


We use tool 5 and tool 1 to write

$$
\begin{aligned}
\left|\Delta t B_{6}^{n}\right| & \leq c_{0}\left\|E_{p}^{A, n}\right\|_{L^{2}}\left(\Delta t \int_{t^{n-1}}^{t^{n}}\left\|\partial_{t} E_{p}^{I}\right\|_{L^{2}}^{2}\right)^{\frac{1}{2}} \\
& \leq \frac{\varepsilon \Delta t}{2}\left\|E_{p}^{A, n}\right\|_{L^{2}}^{2}+\frac{c_{0}^{2}}{2 \varepsilon} \int_{t^{n-1}}^{t^{n}}\left\|\partial_{t} E_{p}^{I}\right\|_{L^{2}}^{2}
\end{aligned}
$$

and we set $\varepsilon=\frac{1}{4}$ for $n=N$ and $\varepsilon=\frac{\varepsilon_{r}}{4}$ for the others $n$ leading to

$\left|2 \Delta t \sum_{n=1}^{N} B_{6}^{n}\right| \leq \frac{\varepsilon_{r}}{4} \sum_{n=1}^{N-1} \Delta t\left\|E_{p}^{A, n}\right\|_{L^{2}}^{2}+\frac{\Delta t}{4}\left\|E_{p}^{A, N}\right\|_{L^{2}}^{2}+\frac{4 c_{0}^{2}}{\tilde{\varepsilon}_{r}}\left\|\partial_{t} E_{p}^{I}\right\|_{L^{2}\left(0, T ; L^{2}\right)^{2}}^{\square}$ 